NATIONAL LABORATORY

MANAGED BY UT-BATTELLE

FOR THE DEPARTMENT OF ENERGY

\title{
Materials and Configuration from NMIS-Type Neutron Imaging and Gamma Spectroscopy
}

May 2012

Prepared by

A. L. Swift

$\frac{\text { பT-BATTELLE }}{\text { ORNL-27(4.00) }}$ 


\section{DOCUMENT AVAILABILITY}

Reports produced after January 1, 1996, are generally available free via the U.S. Department of Energy (DOE) Information Bridge.

Web site http://www.osti.gov/bridge

Reports produced before January 1, 1996, may be purchased by members of the public from the following source.

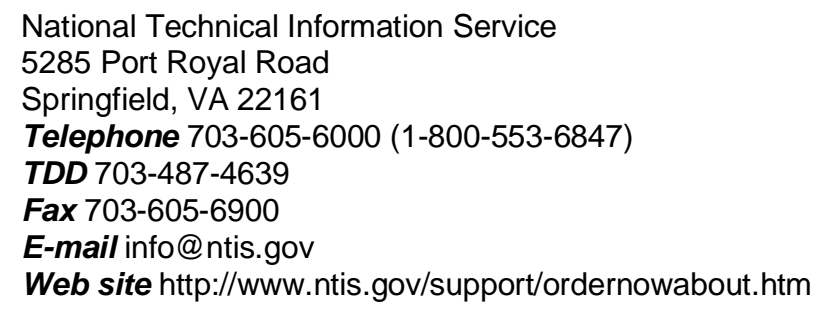

Reports are available to DOE employees, DOE contractors, Energy Technology Data Exchange (ETDE) representatives, and International Nuclear Information System (INIS) representatives from the following source.

Office of Scientific and Technical Information

P.O. Box 62

Oak Ridge, TN 37831

Telephone 865-576-8401

Fax 865-576-5728

E-mail reports@osti.gov

Web site http://www.osti.gov/contact.html

This report was prepared as an account of work sponsored by an agency of the United States Government. Neither the United States Government nor any agency thereof, nor any of their employees, makes any warranty, express or implied, or assumes any legal liability or responsibility for the accuracy, completeness, or usefulness of any information, apparatus, product, or process disclosed, or represents that its use would not infringe privately owned rights. Reference herein to any specific commercial product, process, or service by trade name, trademark, manufacturer, or otherwise, does not necessarily constitute or imply its endorsement, recommendation, or favoring by the United States Government or any agency thereof. The views and opinions of authors expressed herein do not necessarily state or reflect those of the United States Government or any agency thereof. 
Global Nuclear Security Technology Division

MATERIALS AND CONFIGURATION FROM NMIS-TYPE NEUTRON IMAGING AND GAMMA SPECTROSCOPY

\author{
A. L. Swift
}

Date Published: May 2012

Prepared by

OAK RIDGE NATIONAL LABORATORY

Oak Ridge, Tennessee 37831-6283

managed by

UT-BATTELLE, LLC

for the

U.S. DEPARTMENT OF ENERGY

under contract DE-AC05-00OR22725 



\section{CONTENTS}




APPENDIX A. PROCESS FOR THE ANAL YSIS OF NMIS TYPE NEUTRON IMAGING AND GAMMA SPECTROSCOPY.

APPENDIX B. ESTIMATION OF URANIUM ENRICHMENT FROM 186-1001 KEV GAMMA LINES

APPENDIX C. ESTIMATION OF URANIUM ENRICHMENT FROM 186-258 KEV GAMMA LINES 


\section{LIST OF FIGURES}

Figure

Page

1 Diagram of the D-T generator used by NMIS for active interrogation (units are in

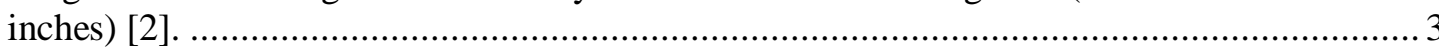

In typical NMIS measurement, an unknown target is interrogated by a D-T neutron generator with an associated alpha-particle detector. 3 Diagram of the eight induced fission radiation detectors in a two-row array (dark brown) with plastic shielding (light brown) between them to decrease cross talk. ................. 5 Illustration of the D-T source (yellow) whose center pixel is perpendicular to the HPGe

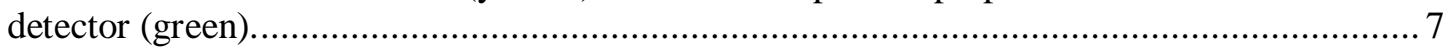

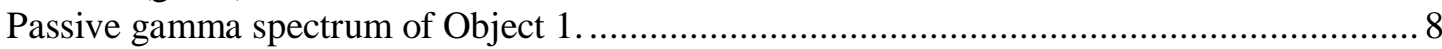

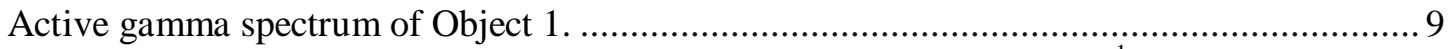

FBP vertical scan of Object 1 that shows the attenuation coefficient $\left(\mathrm{cm}^{-1}\right)$ as a function of height $(\mathrm{cm})$ and detector slot.

Significant counts in the passive gamma spectra and their energies that indicate the presence of uranium isotopes (in keV): 186, 258, 511 (pair production peak), 742, 766, 786, 1001, and 1510.

Significant counts in the active gamma spectra and their energies (in keV): 511 (pair production peak), 847, 1201 (double escape peak), 1712 (single escape peak), 2223, 3416 (double escape peak), 3684, 3927 (single escape peak), 4438, and 4945................... 13

Vertical scan of Object 1.

Attenuation lengths $(\mu x)$ of Object 1 plotted against detector slot (correlates to lateral position within the object) for a single rotation....

FBP radial tomograph of Object 1 that plots the attenuation coefficient $\left(\mathrm{cm}^{-1}\right)$ as a function of distance $(\mathrm{cm})$.

Top: The TAKE process iterated between the measured attenuation length curve (blue) and one that is produced by forward-projecting the guessed object dimensions onto the detectors (green)......

Initial sinogram (left) and the reconstructed sinogram (right) used to create the MLEM tomograph of Object 1.

Reconstructed tomographs at 1, 5, 20, and 50 iterations for Object 1. MLEM tomograph (left) of Object 1 at iteration 50 with adjusted $x$ and $y$ dimensions to show boundary positions.

Fission mapping plots for single (left) and double (right) coincidence counts for the reference data.

Feynman variance of the reference data plotted against the alpha pixel number to allow for a visualization of the multiplication seen by each pixel.

Singles MLEM reconstruction of the object's sinogram (left) and tomograph (right) after 50 iterations.

Doubles MLEM reconstruction of the object's sinogram (left) and tomograph (right) after 50 iterations.

21 Overlay of the MLEM tomograph reconstruction for singles (left) and doubles (right) coincidence data.

22 Centerline slice of the MLEM fission reconstruction doubles tomograph with the two peaks corresponding to the cylindrical region of HEU shielded on the outside by a region of DU. MCNP-PoliMi geometry showing the material regions: void (white), HEU (blue), DU (red), and polyethylene (dark red). 
24 Pixel 9 TOF plot of the total singles counts vs time for the four MCNP-PoliMi models (red, black, green, and pink) and for the reference data (blue)...

25 Pixel 9 enrichment curve generated from the integral of the four MCNP-PoliMi models' TOF plots from 35 to 80 ns.

Illustration of the interpolation method equivalent to the quadratic formula used to determine the enrichment of the HEU region of Object 1. 


\section{LIST OF TABLES}

Table

Page

$1 \quad$ Uranium isotopes in the passive gamma spectra of Object 1 .......................................... 11

$2 \quad$ Nuclide peak energies for the net active gamma spectra ........................................... 12

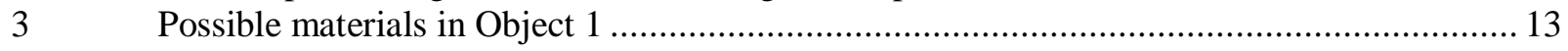

$4 \quad$ Region boundaries and overall heights of the material regions of Object 1 as determined by Mark Walker ........................................................................................... 14

$5 \quad$ Visual inspection and TAKE radial dimensions and attenuation coefficients for

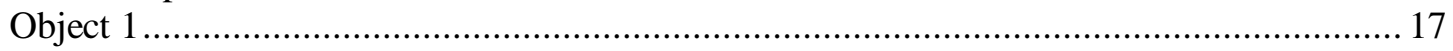

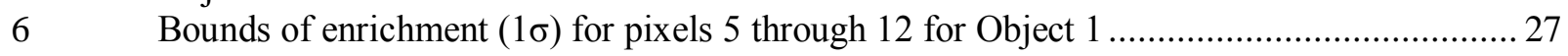

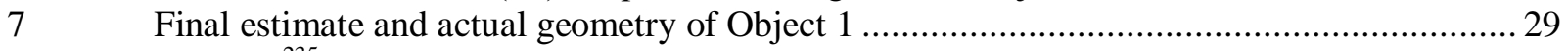

$8 \quad$ Mass of ${ }^{235} \mathrm{U}$ in the final estimate and actual geometry of Object 1 ................................... 29

$9 \quad$ Percent difference between final estimate and actual geometry of Object 1 ........................ 29 



\section{ABBREVIATED TERMS}

$\begin{array}{ll}\text { DOE } & \text { US Department of Energy } \\ \text { D-T } & \text { deuterium-tritium } \\ \text { DU } & \text { depleted uranium } \\ \text { FBP } & \text { filtered back projection } \\ \text { FNMIS } & \text { Fieldable Nuclear Material Identification System } \\ \text { GADRAS } & \text { Gamma Detector Response and Analysis Software } \\ \text { HEU } & \text { highly enriched uranium } \\ \text { HPGe } & \text { high-purity germanium } \\ \text { INL } & \text { Idaho National Laboratory } \\ \text { MCA } & \text { multichannel analyzer } \\ \text { MLEM } & \text { maximum likelihood expectation maximization } \\ \text { NMIS } & \text { Nuclear Material Identification System } \\ \text { ORNL } & \text { Oak Ridge National Laboratory } \\ \text { PMT } & \text { photomultiplier tube } \\ \text { TOF } & \text { time of flight } \\ \text { YAP:CE } & \text { cerium-doped yttrium aluminum perovskite }\end{array}$





\section{ACKNOWLEDGMENTS}

I would like to acknowledge the following Oak Ridge National Laboratory and/or University of Tennessee staff:

Dr. Jason Hayward and Dr. John Mihalczo for their continued support and guidance

Dr. Brandon Grogan for his hard work generating the simulated data necessary for this project

Dr. Howard Hall for his mentorship as a committee member

Dr. Seth McConchie, Dr. James Henkel, Dr. Jim Mullens, Dr. Paul Hausladen, Dr. Eric Sword, and Mark Walker for additional help with this project

I would also like to acknowledge the US Department of Energy for supporting this work at Oak Ridge National Laboratory. 



\begin{abstract}
The Nuclear Material Identification System (NMIS) was first developed at Oak Ridge National Laboratory (ORNL) by the US Department of Energy (DOE) in 1984 for nuclear material control and accountability. Since the mid-1990s, NMIS has been sponsored by the DOE/National Nuclear Security Administration Office of Nuclear Verification for possible future treaty verification applications. NMIS is being developed into a fieldable form (designated FNMIS) and will incorporate gamma spectroscopy and an information barrier. This project was supported by DOE at ORNL. Its purpose was to validate a procedure intended for future use in NMIS. The procedure was developed to determine the geometry and composition of an object from data obtained by neutron imaging, induced fission radiation detection, and passive and active gamma spectroscopy. In the test, the procedure was applied to simulated data that were produced by the PoliMi and MCNPX (version 2.6.0) modeling software. The simulation was based on the description of an object that was not known to the investigator. The investigator's goal was to accurately reproduce the description of the object by applying the procedure in question to the simulated data. Gamma spectroscopy showed that polyethylene was on the outside and that depleted uranium (DU) metal was inside the polyethylene. Analysis of fast neutron imaging data determined estimates of the radii and heights of the materials and the presence of a central void. Analysis of the fission mapping data showed that highly enriched uranium (HEU) metal was inside the DU. Since transmission imaging cannot distinguish DU from HEU metal, fission mapping was used to determine the boundary between the HEU and the DU. Models created from the simulated data were iterated upon by varying the enrichment of the HEU until the calculated time distribution of coincidences agreed with the simulations. The best approximation indicated that the unknown object was composed of concentric cylinders: a void inside HEU (84.7 wt \% ${ }^{235} U$ ), surrounded by DU, surrounded by polyethylene. The final estimation had the correct materials and geometry, with error in the radius estimates of material regions varying from $1.58 \%$ at best and $4.25 \%$ at worst; error in the height estimates varied from $2 \%$ to $12 \%$. The error in the HEU enrichment estimate was 5.9 wt \%, or within $2.5 \sigma$ of the true value. The accuracies of the determinations could be adequate for arms control applications. Future work will apply this iterative reconstructive procedure to other unknown objects to further test and refine it.
\end{abstract}





\section{INTRODUCTION}

The Nuclear Material Identification System (NMIS) has been under development at Oak Ridge National Laboratory (ORNL) since 1984, sponsored initially by the US Department of Energy (DOE) for nuclear material control and accountability applications and then by the DOE/National Nuclear Security Administration Office of Nuclear Verification in the mid-1990s for possible future treaty verification applications [1]. As a result, it can be used in other nuclear nonproliferation applications, such as template matching for confirmation of inventories of weapons components [2]. NMIS previously used a timetagged californium $\left({ }^{252} \mathrm{Cf}\right)$ spontaneous fission source but most recently has employed an associatedparticle neutron generator with an embedded alpha detector to tag in both time and direction a portion of the emitted neutrons from the deuterium-tritium (D-T) reaction [3]. A fieldable NMIS (designated FNMIS) is being developed to facilitate the future incorporation of gamma spectroscopy and an information barrier [2].

This project, supported by DOE at ORNL, used Monte Carlo-simulated NMIS time coincidence distributions, fast neutron imaging, fission mapping, and passive and active time-tagged gamma ray spectrometry to analyze "Object 1," having an unknown configuration and containing both fissile and nonfissile material. The simulated NMIS neutron data were obtained with MCNP-PoliMi [4]; the gamma ray spectrometry data were modeled with MCNPX version 2.6.0 [5]. The simulated data were presented for analysis to the author, who had no knowledge of Object 1 , to determine its configuration and materials through a previously determined process [6]. As such, it was a blind study to determine how much information could be obtained from each step in the procedure (as well as collectively) and to estimate the configuration of Object 1. The estimate was then modeled with MCNP-PoliMi, analyzed, and compared to the provided time distribution of coincidence data.

This paper is divided into three main sections: a description of the NMIS measurement system, details of the simulation methods, and a description of the analysis process applied to the simulated data. It concludes with the best estimate of the configuration of materials in Object 1 and recommendations for future analyses of unknown objects. The steps in the procedure are given in Appendix A. 



\section{NMIS MEASUREMENT SYSTEM}

The NMIS measurement system has six main components: a D-T generator with an associated alpha detector, imaging detectors, induced fission radiation detectors, the electronics necessary to condition the timing signals, a rotation apparatus (useful for objects without symmetry, such as cuboids), and a highspeed (up to $1 \mathrm{GHz}$ ) processor to compute the time correlation in live time.

\subsection{D-T GENERATOR}

NMIS uses a portable D-T generator (Thermo-Fisher Scientific API120) that employs the following reaction to generate $14.1 \mathrm{MeV}$ neutrons for active interrogation of an unknown object $[6,8]$ :

$$
{ }_{1}^{2} \mathrm{H}+{ }_{1}^{3} \mathrm{H} \rightarrow{ }_{0}^{1} \mathrm{n}+{ }_{2}^{4} \mathrm{He}
$$

This neutron energy is desirable due to its ability to more efficiently pass through materials containing hydrogen [2]. Approximately $4 \times 10^{7}$ neutrons per second are produced isotropically in the current NMIS generators [9]. That rate is achieved by aiming a beam composed of deuterons and tritons (less than $60 \mu \mathrm{A}$ ) at a $5 \mathrm{~mm}$ diameter zirconium target embedded with tritium and deuterium atoms (Fig. 1).

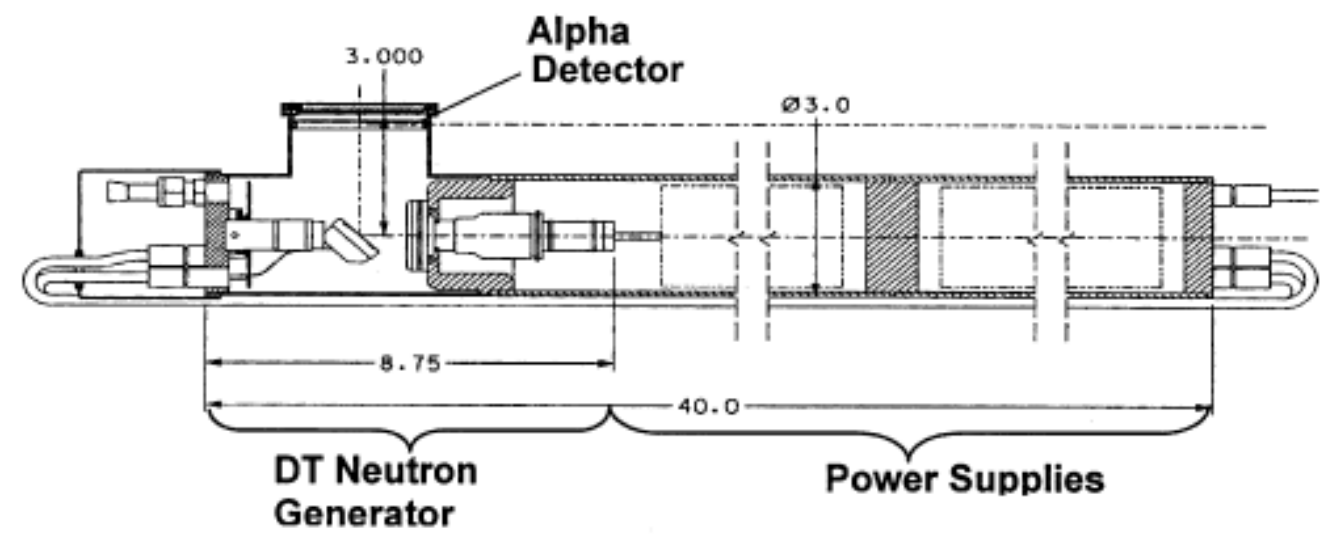

Fig. 1. Diagram of the D-T generator used by NMIS for active interrogation (units are in inches) [2].

The D-T reaction emits neutrons and 3.5 MeV alphas traveling in almost opposite directions of the neutrons [1]. The generator has an associated alpha detector [a cerium-doped yttrium aluminum perovskite (YAP:Ce) scintillator] to define the direction of the alpha particle and thus to determine the direction of the associated neutron. This is necessary for the coincidence counting used in neutron imaging and fission mapping. Alpha particles entering the YAP:Ce scintillator produce light, which is transferred through a fiber-optic face plate to a Hammamatsu H9500 photomultiplier tube (PMT) on the outside of the generator. The PMT, coupled to the outside of the fiber-optic faceplate, uses a row of 16 pixels to electronically collimate the neutron beam into a corresponding 16 pixel horizontal fan beam. At that point, the pixilated fan beam then passes through the interrogation object and reaches the detectors on the other side, all of which can be seen in Fig. 2 [6].

The alpha detection system serves the dual use of time-tagging and directionally tagging the neutrons, which is necessary for the coincidence counting used in imaging the unknown object. Because the neutron energy is known, the time of arrival of the $14.1 \mathrm{MeV}$ neutrons at the detector is known, and the counts in an approximately $5 \mathrm{~ns}$ time window at that time are used for transmission imaging [9]. Scattered radiation and induced fission radiation usually arrive at the detectors at a later time. 


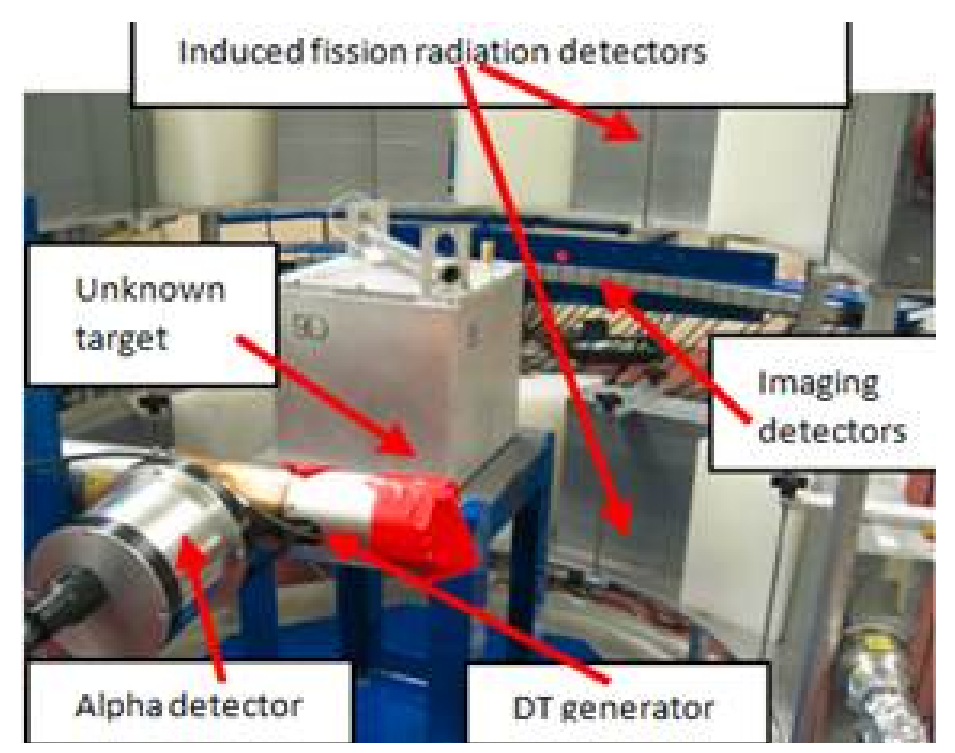

Fig. 2. In typical NMIS measurement, an unknown target is interrogated by a D-T neutron generator with an associated alpha-particle detector. Scattered and fission neutrons are then detected by respective imaging and induced fission radiation detectors on the far side of the object.

\subsection{IMAGING DETECTORS}

NMIS uses thirty-two $2.54 \times 2.54 \times 10.16 \mathrm{~cm}$ plastic EJ-200 scintillators as the imaging detectors, arrayed along an arc with a $115 \mathrm{~cm}$ radius with respect to the neutron production spot in the D-T generator [9]. In a process known as subsampling, the imaging detector arc is shifted laterally by some fraction (usually one-fourth) of the distance between the imaging detectors to increase the spatial resolution. At one-fourth the original distance, a total of 128 detector positions (or four locations per detector) were simulated [7]. If two counts occur in adjacent detectors within a preselected time window, the second count is ignored so that the possibility of counting a single neutron twice is eliminated.

The imaging detector arc can also be raised or lowered to perform measurements at set increments along the height (z-axis) of the object to account for the possibility of nonuniformity in the geometry or in the composition of the object. Since NMIS contains a rotation mechanism on which the object is placed, the object can also be rotated at each height increment in the $x-y$ plane. The imaging data gathered at each rotation is then combined to form a sinogram, which is a plot of the detector measurement position vs the detector angular position and from which a tomograph of the object can be reconstructed. For cylindrically symmetric objects with a vertical axis of symmetry, the rotation is not necessary if location with respect to the source is known.

\subsection{INDUCED FISSION RADIATION DETECTORS}

For these simulations and analyses, NMIS employed eight $25 \times 25 \times 8 \mathrm{~cm}$ induced fission radiation detectors that are set in a two-tier aluminum array so that four detectors sit above the imaging detectors and four sit below (see Figs. 2 and 3). The proton recoil scintillators are separated by 6 in. of polyethylene to reduce cross talk between detectors [10]. For this work, the fission detectors were in an arc radius of $50 \mathrm{~cm}$ from the center of Object 1 [11]. The detectors are larger than the imaging detectors to detect the induced fission radiation, which is usually an order of magnitude less than the transmission radiation. The induced fission radiation detectors are used for fission mapping and serve the purpose of measuring multiplicity (i.e., the number of single and double neutrons resulting from induced fission in the object). 
That information can be used to determine the enrichment levels of fissile material in an interrogation object because there is a direct correlation between the number of fission neutrons detected (singles, doubles, and so on) and the amount of fissile material present [7].

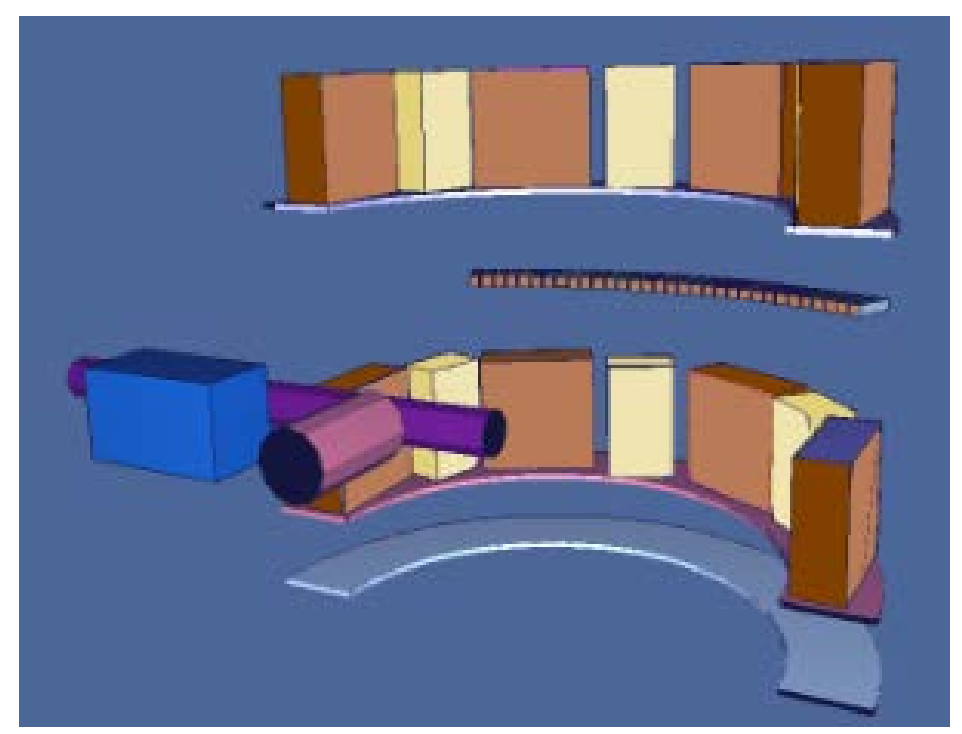

Fig. 3. Diagram of the eight induced fission radiation detectors in a two-row array (dark brown) with plastic shielding (light brown) between them to decrease cross talk.

The thirty-two imaging detectors can be seen in the center (brown), with the D-T generator shown at left in blue and purple and its associated alpha-particle detector shown in dark pink [7].

To accomplish fission mapping, NMIS uses source-triggered, random, and time-gated multiplicities (e.g., singles, doubles, triples). The time interval is selected to maximize the probability that detected neutrons are indeed from fission and are not from other sources such as inelastic gamma rays. The window begins slightly after the radiation induced by the interrogation neutrons reaches the detectors and ends when the last fission chain neutrons reach the induced fission radiation detectors. In theory, the gamma rays from the initial induced fissions have already passed through the detectors by the time fission neutrons arrive at the detectors. However, prompt gamma rays from fissions later in the fission chain are detected in the time window. Additional gamma ray reductions can be achieved by pulse shape discrimination with liquid scintillators; plastic scintillators can only use timing to discriminate between photons and neutrons [7]. (Pulse shape discrimination was not used in the investigation of Object 1.)

\subsection{ELECTRONICS SYSTEM AND DATA PROCESSING}

After digitization, all signals from all detectors go to the NMIS high-speed data acquisition processor via shielded cables. The data acquisition processor computes time correlations online between the 16 alpha pixels and each imaging detector in the array and between the induced fission radiation detectors in 1 ns increments [1]. All correlations can be normalized to the source strength through dividing the number of counts in each time interval by the total counts recorded in that pixel over the entire measurement [1,12]. (An in-depth look at the capability of the processor is given in Ref. 1.) For gamma spectroscopy, NMIS uses a multichannel analyzer. 



\section{DESCRIPTION OF SIMULATIONS}

This chapter describes the simulated data that were generated at ORNL (B. Grogan, personal communication).

\subsection{SIMULATED EXPERIMENTAL CONFIGURATION}

Object 1 was placed on a stainless steel (SS304) table for both gamma and neutron measurements. The table had dimensions of $45.72 \times 45.72 \times 1.27 \mathrm{~cm}$ and was at a height of $56 \mathrm{~cm}$ above a concrete floor. Passive and active gamma spectroscopy simulations were conducted with MCNPX version 2.6.0. The idealized simulated high-purity germanium (HPGe) gamma detector was a 3 in. cube placed adjacent to ( $1 \mathrm{~mm}$ from the surface of the object to minimize simulation time) and $90^{\circ}$ to the central pixel of the DT beam (Fig. 4).

For neutron imaging and fission mapping, the source-to-center distance was $35 \mathrm{~cm}$ and the source-toimaging-detector distance was $115 \mathrm{~cm}$. Data for 60 rotations of $6^{\circ}$ each were simulated at a height of 70 $\mathrm{cm}$ above the floor; that height was selected because a vertical scan of Object 1 indicated that $70 \mathrm{~cm}$ was the height of the region of interest for a detailed internal

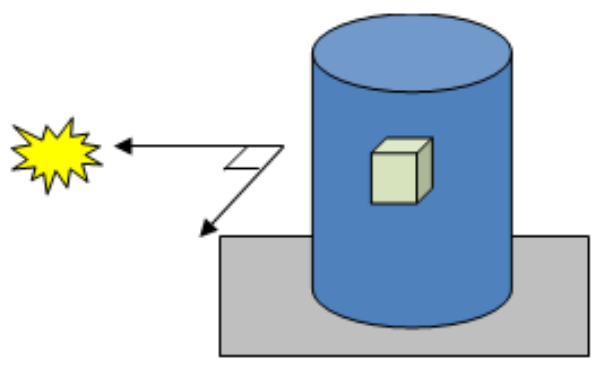

Fig. 4. Illustration of the D-T source (yellow) whose center pixel is perpendicular to the HPGe detector (green).

An interrogation object (blue) and table (gray) are also shown.

slice, or radial tomograph. Because Object 1 was radially symmetric, only the scan obtained at a height of $70 \mathrm{~cm}$ was provided and the sum of all rotations was used in the analysis. In contrast, scans at multiple heights and individual rotations must be used in a measurement of an asymmetric object (for instance, a cuboid). All simulated time correlation data (specifically, transmission, scattered, and neutrons and gammas from fission) were provided by MCNP-PoliMi.

\subsection{SIMULATED GAMMA SPECTROSCOPY DATA}

\subsubsection{Overview of the Gamma Spectroscopy Modeling}

MCNPX version 2.6.0 was used to create the passive and active gamma spectra of Object 1. Detector live time was set to $900 \mathrm{~s}$ for both simulations, and a normalized count was obtained via the following two-step process. First, a pulse height (F8) tally in the HPGe detector was used to record the amount of energy deposited in the detector cell per particle history. Next, that value was converted to gamma counts by multiplying by the live time, the specific activity (or specific activity plus the neutron source rate if an active measurement), the total mass, and a solid angle efficiency correction. After total counts were determined, energy spreading was added to the data by assuming an energy resolution of $0.5 \%$ at the $661 \mathrm{keV}\left({ }^{137} \mathrm{Cs}\right)$ peak and that the resolution everywhere else varied from that value by the square root of the energy.

\subsubsection{Passive Gamma Spectroscopy Data}

The passive gamma ray data were obtained without an interrogation source and are a measure of the photons naturally emitted by Object 1 (e.g., radioactive decay). Physics modeling (algorithms written to model actual detector physics) was used to account for both actual energy deposition in a detector and energy broadening, resulting in features such as photopeaks, Compton edges, and escape peaks. The 
passive spectrum is shown in Fig. 5. When the net gamma spectrum was generated, a natural background count was not simulated because it would be subtracted in practice and because it could vary considerably, depending on the environment.

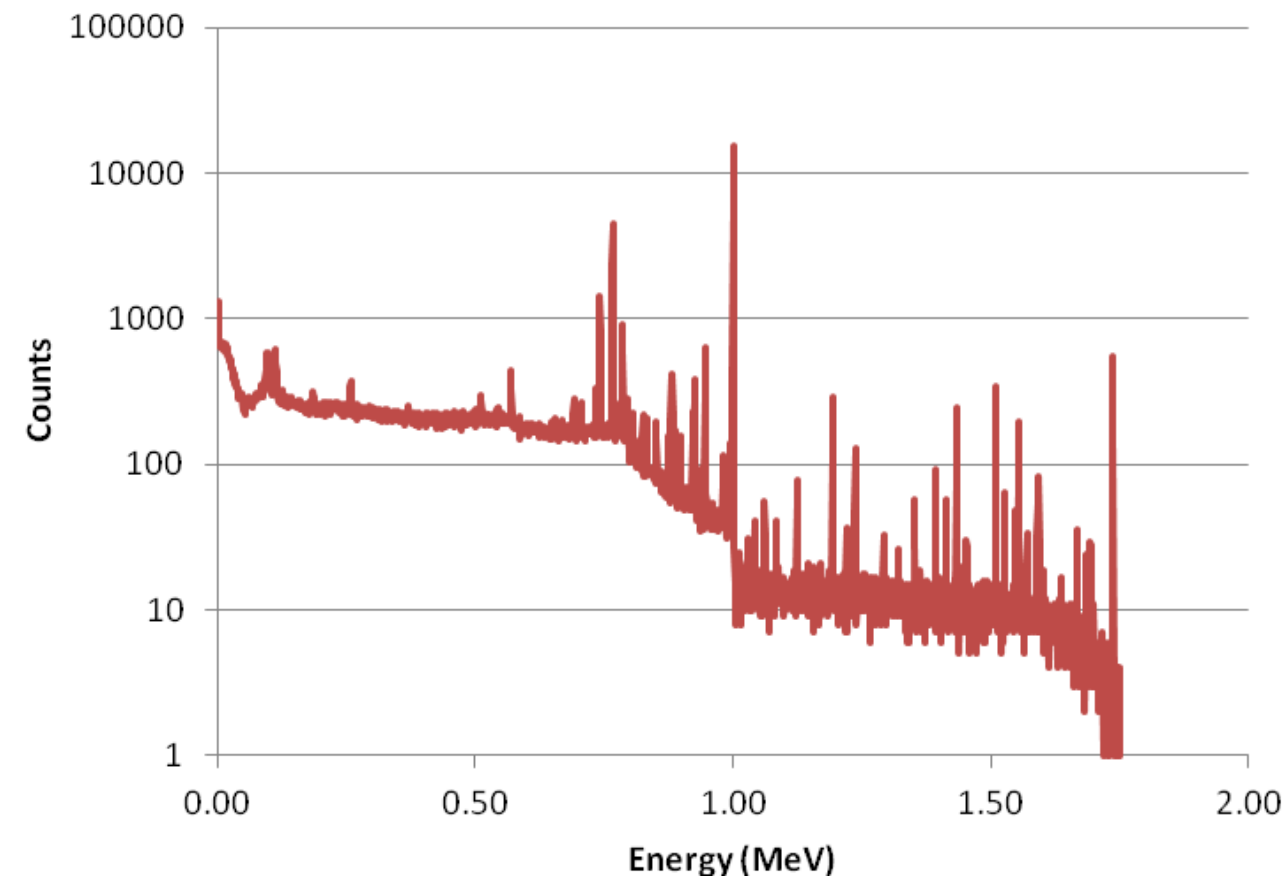

Fig. 5. Passive gamma spectrum of Object 1.

\subsubsection{Active Gamma Spectroscopy Data}

The active gamma ray data were simulated with an interrogation source (14.1 MeV neutrons from the D-T generator). The F8 tally had a $6 \mathrm{MeV}$ cutoff energy and the same physics modeling as the passive gamma data (Fig. 6). The active simulation included the passive data, which would be measured and subtracted in practice. Additional peaks seen in the active gamma data are a result of $(n, g)$ and $\left(n, n^{\prime}\right)$ neutron interactions in the object.

It was important to know what external materials were close to Object 1 for the active simulation because they might also generate capture gamma peaks and may therefore create false positives in the data. The external objects included the stainless steel (SS304) turntable upon which Object 1 was located, an aluminum detector measurement stand, the concrete walls and floor, and lead shielding for the induced fission radiation detectors. 


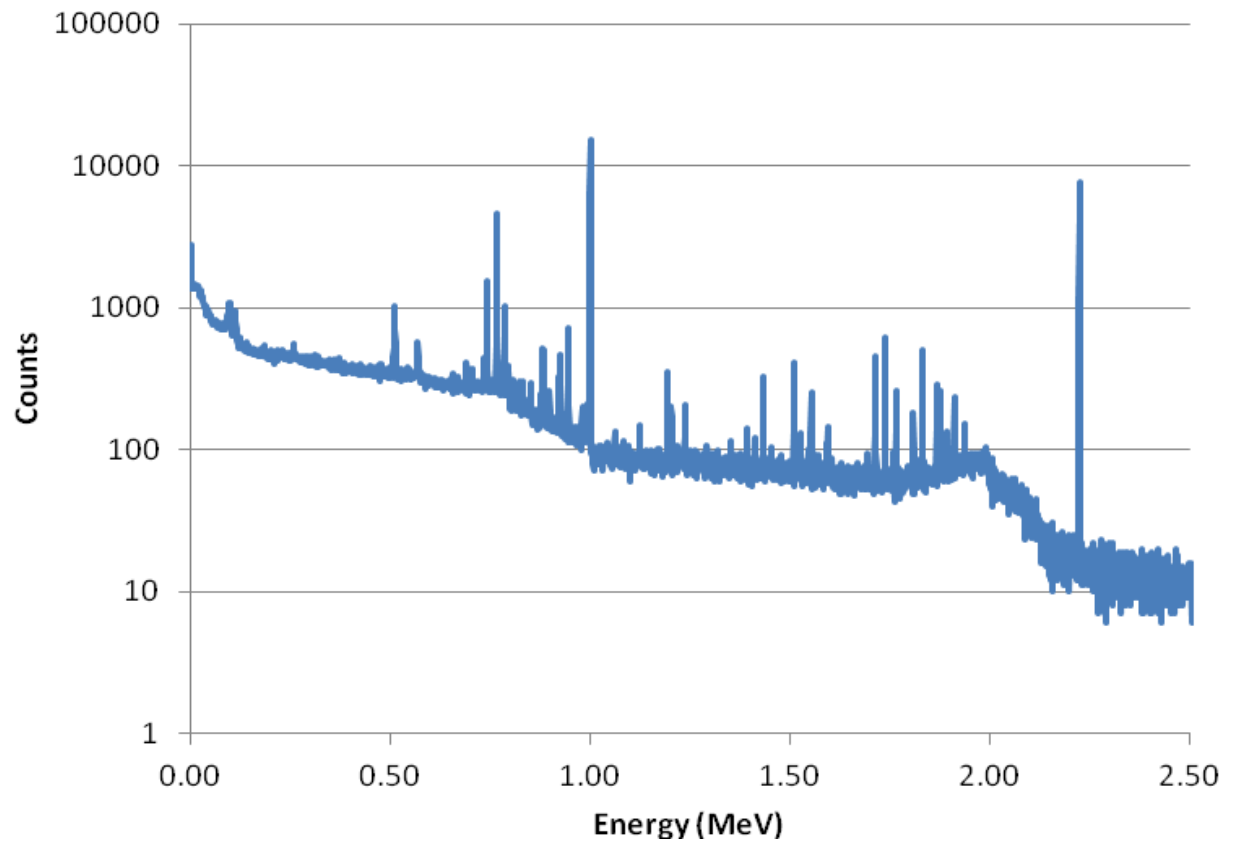

Fig. 6. Active gamma spectrum of Object 1.

\subsection{SIMULATED NEUTRON IMAGING AND FISSION SITE MAPPING DATA}

\subsubsection{Material Attenuation Theory and Transmission Imaging}

Material attenuations are described by Eq. (1),

$$
I=I_{o} e^{-\mu x},
$$

where $I$ is the attenuated flux of neutrons (determined with the object present), $I_{o}$ is the initial flux of neutrons (determined without the object present), $\mu$ is the material-dependent attenuation coefficient for 14.1 MeV source neutrons, and $x$ is the neutron path length (in centimeters) [12]. Equation (1) is used as the basis for the two NMIS neutron image reconstruction techniques, filtered back projection (FBP) (see Sect. 4.2.2) and maximum likelihood expectation maximization (MLEM) iterative reconstruction (see Sect. 4.2.3). A vertical scan of the object is always completed first to determine at what height to create the detailed transmission image (see Sect. 3.3.2). It was determined from a vertical scan of Object 1 that the scan for a transmission image be conducted at $70 \mathrm{~cm}$.

The FBP and MLEM iterative reconstruction methods that were employed to determine the configuration of Object 1 used the same data generated with MCNP-PoliMi. A void simulation was performed with no object present $\left(I_{o}\right)$, followed by a simulation with the object present $(I)$. Induced fission radiation detector simulations were run separately from transmission imaging detector simulations.

\subsubsection{Material Attenuation Theory and Transmission Imaging}

The vertical scan of Object 1 was simulated by modeling the simultaneous movement of the D-T generator and detectors along the height of the object in $1 \mathrm{~cm}$ intervals ranging from 54 to $83 \mathrm{~cm}$ above the floor (Fig. 7). The vertical scan was necessary because spheres and cylinders of the same outer dimension appear the same in radial tomography in a plane at the height corresponding to the largest 
horizontal dimension, whereas a vertical scan easily discriminates between these two geometries. A vertical scan also determines heights of the material regions in Object 1.

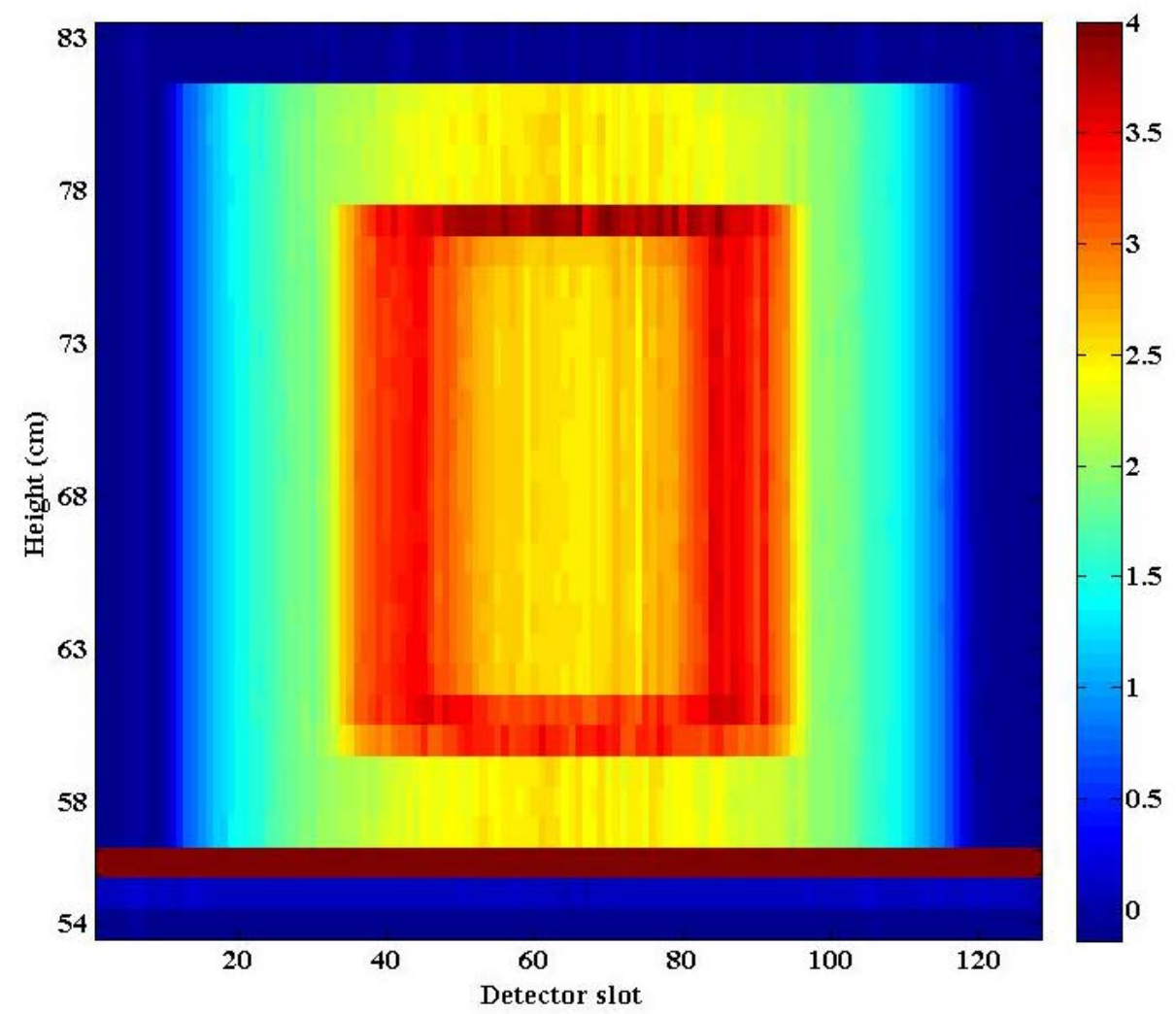

Fig. 7. FBP vertical scan of Object 1 that shows the attenuation coefficient $\left(\mathrm{cm}^{-1}\right)$ as a function of height $(\mathbf{c m})$ and detector slot. Red signifies areas of high attenuation and blue areas of low attenuation (the contrast scale measures attenuation lengths through the object).

\subsubsection{Fission Mapping Data}

Many reactions result from neutron interrogation; those of interest with respect to NMIS and this study include (n,f), (n,n'), (n,2n'), and (n,3n'). The higher the fissile mass and enrichment, the greater the amount of singles, doubles, and so on that are emitted and thus are available for detection. Mapping of fission sites is therefore a useful tool in determining the location, mass, and enrichment of fissile material in a sample. It has been used in past measurements at Idaho National Laboratory (INL) with depleted uranium (DU) around highly enriched uranium (HEU) to determine that the HEUwas on the inside. Enriched uranium and DU have nearly the same neutron attenuations for $14.1 \mathrm{MeV}$ neutrons $\left(0.281 \mathrm{~cm}^{-1}\right.$ and $0.279 \mathrm{~cm}^{-1}$, respectively) and cannot be readily distinguished by neutron transmission, making fission mapping a useful diagnostic tool.

The eight induced fission radiation detectors used for fission mapping were modeled in MCNPPoliMi (each detector having a 50 ns dead time and an energy threshold of $1 \mathrm{MeV}$ for neutrons). Each particle history was tracked for up to $256 \mathrm{~ns}$. 


\section{INTERPRETATION OF SIMULATED MEASUREMENT DATA}

This chapter outlines the methods that were used to analyze and interpret the simulated neutron and photon data described in Chap. 3 to form an initial estimate of the configuration of Object 1 . The estimate was then modeled with MCNP-PoliMi, and the data produced by the modeling were compared with the reference simulation data to determine the accuracy of the estimate.

\subsection{GAMMA SPECTROSCOPY DATA}

\subsubsection{Analysis of Passive Gamma Spectroscopy Data}

Analysis of passive gamma spectroscopy data was the first step in the previously developed process of determining the configuration of Object 1 . It was employed to determine what, if any, fissile or fissionable material was present. No peaks indicating the presence of weapons-grade plutonium were found (namely, 375 and $414 \mathrm{keV}$ ), but many peaks indicating the possible presence of HEU or DU (specifically, ${ }^{235} U$ and ${ }^{238} U$ peaks) were present in the passive spectrum [13]. Some of the visible peaks and their respective isotopes are listed in Table 1, and they are labeled on the passive spectrum in Fig. 8.

Another use for the passive gamma spectra was to determine uranium enrichment, $E$. Enrichment was estimated in two ways, the first performed by the author and the second by Dr. Brandon Grogan. The first method for estimating $E$ used a ratio of the ${ }^{235} \mathrm{U}$ to ${ }^{238} \mathrm{U}$ net counts (referred to in this paper as $N_{235}$ and $N_{238}$,

Table 1. Uranium isotopes in the passive gamma spectra of Object 1

\begin{tabular}{cc}
\hline $\begin{array}{c}\text { Uranium } \\
\text { isotope }\end{array}$ & Peak energy (keV) \\
\hline${ }^{235} \mathrm{U}$ & Low-energy X rays $(95,98,111)$ \\
& 186 \\
& $258^{a}$ \\
$511^{b}$ \\
742 \\
${ }^{238} \mathrm{U}$ & 766 \\
& 786 \\
& 1001 \\
& 1193 \\
& 1510 \\
\hline
\end{tabular}

\footnotetext{
${ }^{a}$ Barely visible

${ }^{b}$ Annihilation peak
} respectively) corrected for peak yields [12,13]; net counts were found by selecting regions of interest. Peaks chosen for that purpose were the $186 \mathrm{keV}$ (the result of ${ }^{235} \mathrm{U}$ ) line and the $1001 \mathrm{keV}$ and $258 \mathrm{keV}$ (both the result of ${ }^{238} \mathrm{U}$ ) lines (see Appendix B and Appendix $\mathrm{C}$ for the equations used). The differing effects of attenuation can be mitigated and thus the accuracy of the ratio can be increased by taking the ratio of peaks that are closer together in energy. This is illustrated with the results, as the 186:1001 ratio yielded $E=0.056 \mathrm{wt} \%$ and the 186:258 ratio yielded $E=0.066 \mathrm{wt} \%$; as the peaks get closer in energy, the enrichment approaches $E=0.2 \mathrm{wt} \%$, which is the enrichment of DU. HEU may be present but interior to and shielded by DU; the question of the presence of HEU is evaluated at a later stage in the analysis process documented in this report in Sect. 4.2.6.

The second method employed the Gamma Detector Response and Analysis Software (GADRAS) to estimate $E$. GADRAS uses a multiple-regression iterative algorithm that "employs radiation source and detector response models to predict the response of user-defined detectors to user-defined sources" [15]. Parameters of a simple spherical geometrical model, such as materials and enrichment, were iteratively varied by GADRAS to generate an estimated spectrum to match the simulated passive spectrum and thus predict enrichment. The analysis estimated $E=0.2 \mathrm{wt} \%{ }^{235} \mathrm{U}$, pointing to the presence of DU with less uncertainty than the first method. The GADRAS-based method used the total spectrum, including the low-energy $\mathrm{X}$ rays in the $100 \mathrm{keV}$ region, which is most likely the reason for its increased accuracy because it could then account for differences in attenuation. Thus, it can be concluded from both methodologies in this step that DU is present. 


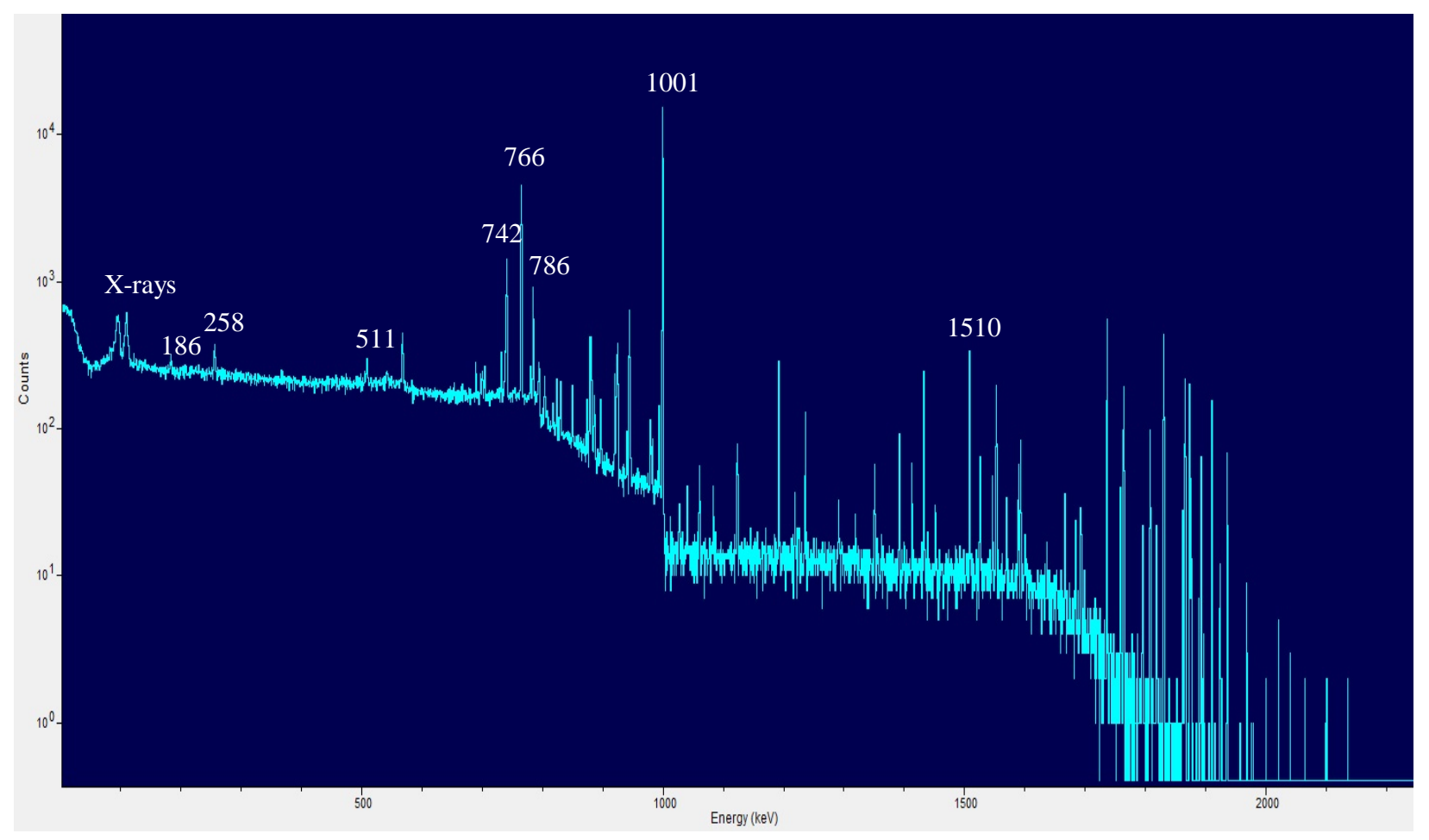

Fig. 8. Significant counts in the passive gamma spectra and their energies that indicate the presence of uranium isotopes (in keV): 186, 258, 511 (pair production peak), 742, 766, 786, 1001, and 1510.

\subsubsection{Analysis of Active Gamma Spectroscopy Data}

Whereas the passive spectrum of the Object 1 was used to determine the presence of fissile material, the active spectrum was used to ascertain the existence of nonfissile material and was the second step in forming an initial estimate of the configuration of Object 1. Significant peak energies and associated single and double escape peaks present in the active spectrum are listed in Table 2 and are labeled on the active spectrum in Fig. 9. In Table 2, iron (n,n') reactions from the associated alpha pixel in the D-T generator in the stainless steel measurement table that are being detected by the HPGe detector directly above the table. As such, the presence of iron in the object is doubtful.

Table 2. Nuclide peak energies for the net active gamma spectra

\begin{tabular}{lcc}
\hline Nuclide & Peak energy (keV) & Interaction mechanism \\
\hline $\mathrm{Fe}$ & 847 & $\left(\mathrm{n}, \mathrm{n}^{\prime}\right)$ \\
& 1201 & Double escape peak \\
$\mathrm{H}$ & 1712 & Single escape peak \\
& 2223 & $(\mathrm{n}, \mathrm{g})$ \\
& 3416 & Double escape peak \\
& 3684 & $(\mathrm{n}, \mathrm{g})$ \\
$\mathrm{C}$ & 3927 & Single escape peak \\
& 4438 & $\left(\mathrm{n}, \mathrm{n}^{\prime}\right)^{b}$ \\
& 4945 & $(\mathrm{n}, \mathrm{g})$ \\
\hline
\end{tabular}

${ }^{a}$ The $\mathrm{C}$ double escape peak from $4945 \mathrm{keV}(\mathrm{E}=3923 \mathrm{keV})$ could not be resolved from this $\left(n, n^{\prime}\right)$ interaction.

${ }^{b}$ The $\mathrm{C}$ single escape peak from $4945 \mathrm{keV}$ ( $\left.\mathrm{E}=4434 \mathrm{keV}\right)$ could not be resolved from this (n,n') interaction. 


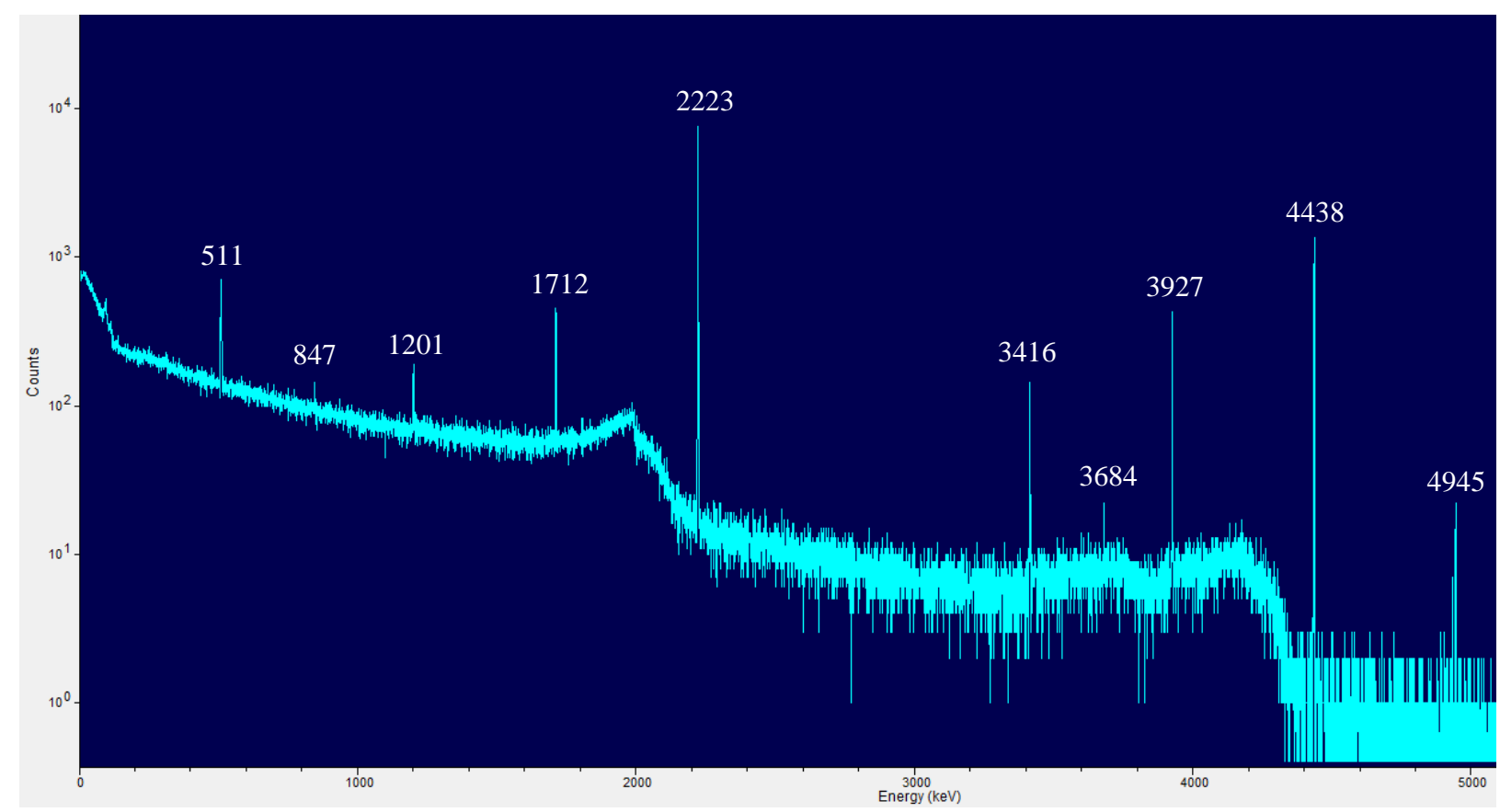

Fig. 9. Significant counts in the active gamma spectra and their energies (in keV): 511 (pair production peak), 847, 1201 (double escape peak), 1712 (single escape peak), 2223, 3416 (double escape peak), 3684, 3927 (single escape peak), 4438, and 4945.

\subsubsection{Formulation of an Initial Estimate of Materials Present in Object 1}

By examining both Table 1 and Table 2, initial estimates were made on the materials in Object 1 . The estimates, along with their corresponding material attenuation coefficients, are listed in Table 3 . This was a difficult task, as some isotopes are indicators of multiple materials. For instance, carbon could stand alone as graphite, or it could be one of the components of polyethylene (the second being hydrogen). Thus potential materials were initially established to be polyethylene, iron, and uranium (either in the forms of DU, HEU, or both). Iron was later ruled out due to (n,n') reactions in the stainless steel measurement table, and graphite was found to be unlikely due to the presence of the hydrogen capture gamma ray, which indicates a hydrocarbon, possibly polyethylene.

Table 3. Possible materials in Object 1

\begin{tabular}{lcc}
\hline Nuclides found & Possible materials & $\begin{array}{c}\text { Attenuation coefficient } \\
\left(\times \mathbf{1 0}^{-2} \mathbf{~} \mathbf{m}^{-\mathbf{1}}\right)\end{array}$ \\
\hline $\mathrm{C}$ & Polyethylene & 10.98 \\
& $\mathrm{DU}$ & 27.90 \\
Uranium and its daughters & $\mathrm{HEU}^{a}$ & 28.14 \\
$\mathrm{H}$ & Polyethylene & 10.98 \\
\hline
\end{tabular}

${ }^{a} \mathrm{DU}$ might be shielding the HEU on the outside and thus HEU might be present. 


\subsection{TRANSMISSION NEUTRON IMAGING DATA}

\subsubsection{Vertical Scan Data}

Completion of a vertical scan of Object 1 was the next step after completion of the analysis of the passive and active gamma spectra. The vertical scan was conducted to determine regions of interest and therefore the best height to complete a radial scan of Object 1 . The optimal height was determined to be the vertical center of the object, or $70 \mathrm{~cm}$. The vertical scan also allowed for the determination of the height of the material regions in Object 1 (Fig. 10). The heights, listed in Table 4, were determined by visual inspection of the image in Fig. 10 at ORNL (M. Walker, unpublished data).

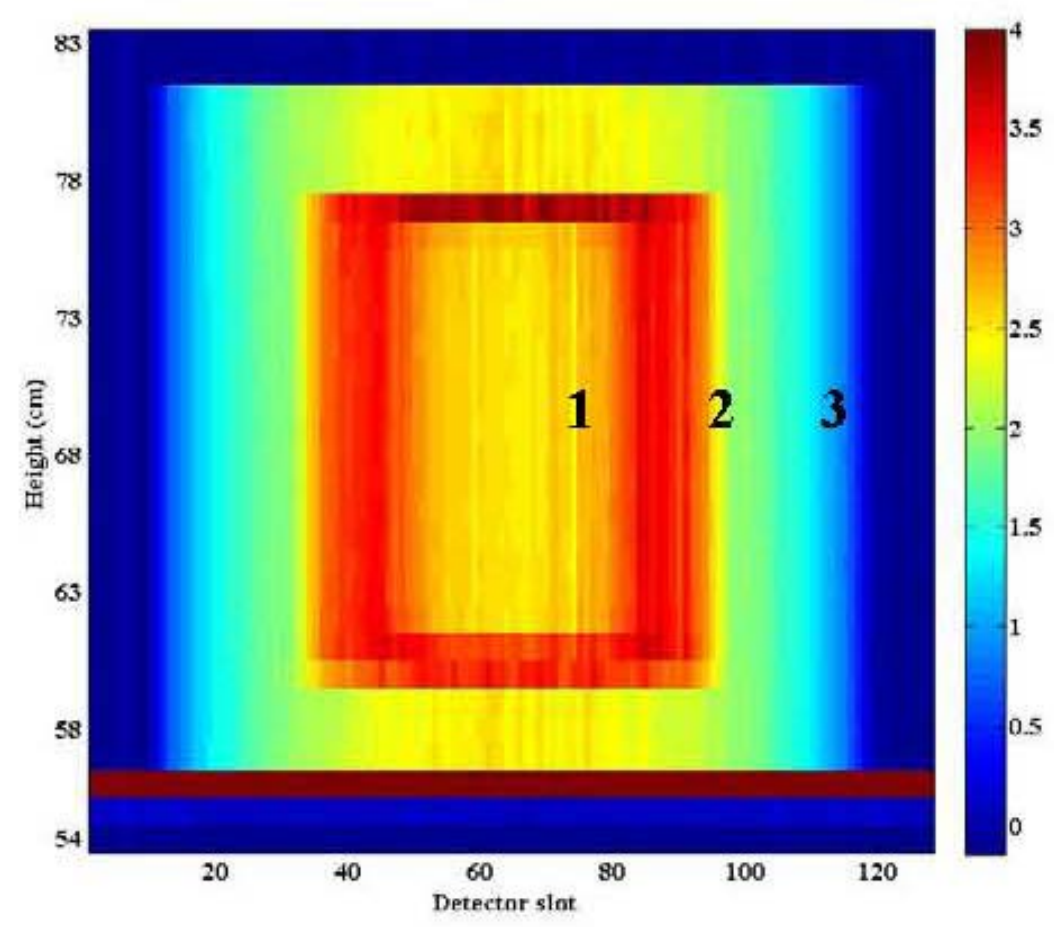

Fig. 10. Vertical scan of Object 1. Red signifies areas of high attenuation, blue, those of low attenuation. Supposed material regions are labeled to match those in Table 4. (Regions analyzed by Mark Walker.)

Table 4. Region boundaries and overall heights of the material regions of Object 1 as determined by Mark Walker

\begin{tabular}{ccc}
\hline Material region & Region boundaries $\mathbf{( c m )}$ & Overall height $\mathbf{( c m )}$ \\
\hline 1 & 60 to 77 & 17 \\
2 & 59 to 78 & 19 \\
3 & 56 to 81 & 25 \\
\hline
\end{tabular}




\subsubsection{Filtered Back Projection}

The next set of steps in the process of determining the materials possibly in Object 1involved the analysis of the transmission neutron imaging data at the centerline. That part of the procedure allowed for the position and geometry of these materials to be established. The FBP technique was the first of two transmission image analyses that were employed for this purpose; the other technique, MLEM, will be described in the following section.

The FBP method used an existing Matlab code to plot the attenuation length $(\mu x)$ against detector lateral position (informally referred to as a detector slot), where each detector slot corresponds to one of detector's four lateral subsample positions (Fig. 11). The material-dependent attenuation coefficient, $\mu$, was next isolated from $\mu x$ by normalizing the attenuation length to the distance that the interaction point was within Object 1 . This could be accomplished because the relative geometry of the source, detectors, and the object were all known variables, allowing for the object's dimensions (and thus $x$ ) to be determined. The attenuation coefficient was then plotted as a function of radial distance by summing all of the sixty rotations and assuming rotational symmetry, thereby forming a radial image of the material regions of Object 1 (Fig. 12).

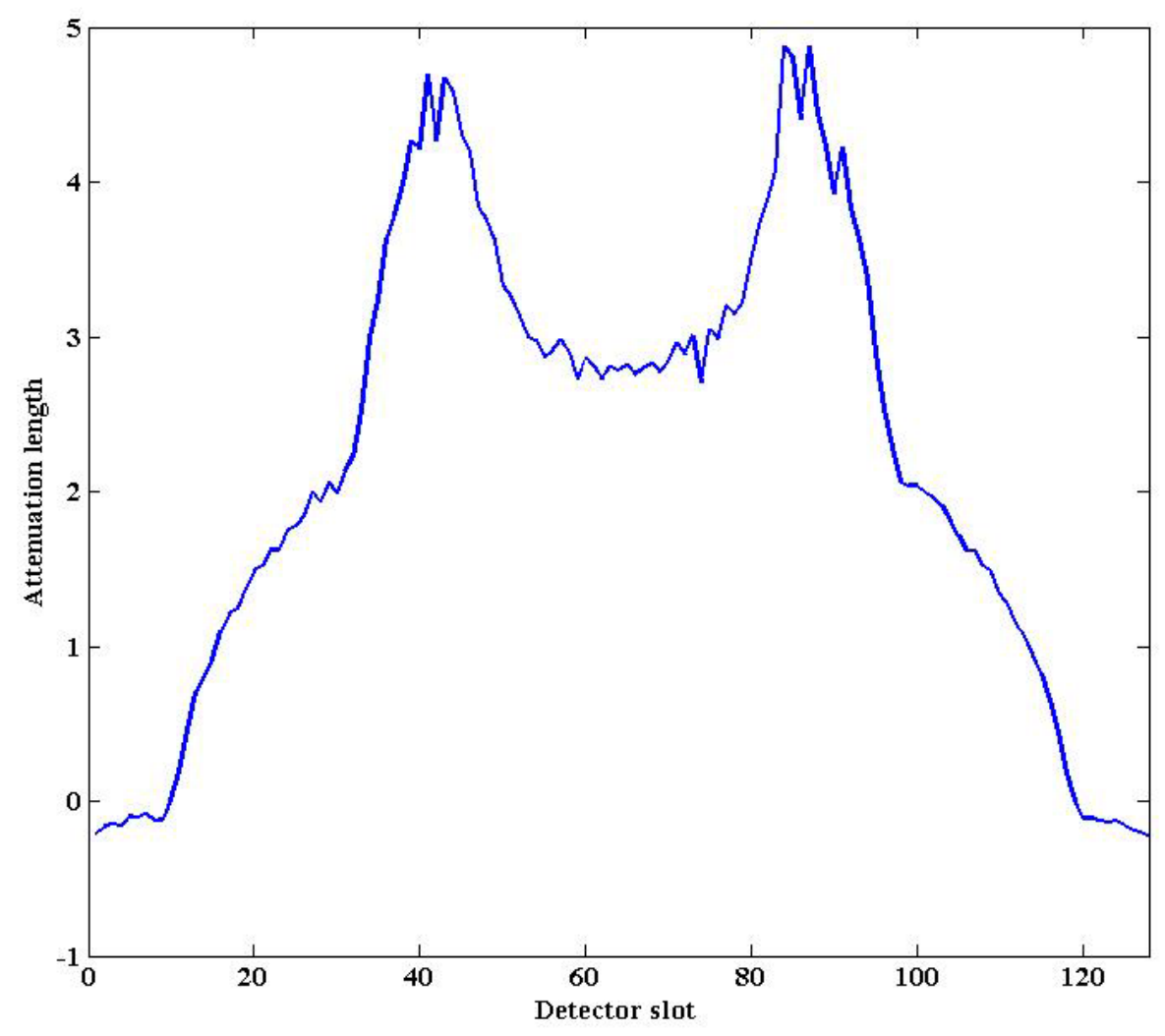

Fig. 11. Attenuation lengths $(\mu x)$ of Object 1 plotted against detector slot (correlates to lateral position within the object) for a single rotation. 


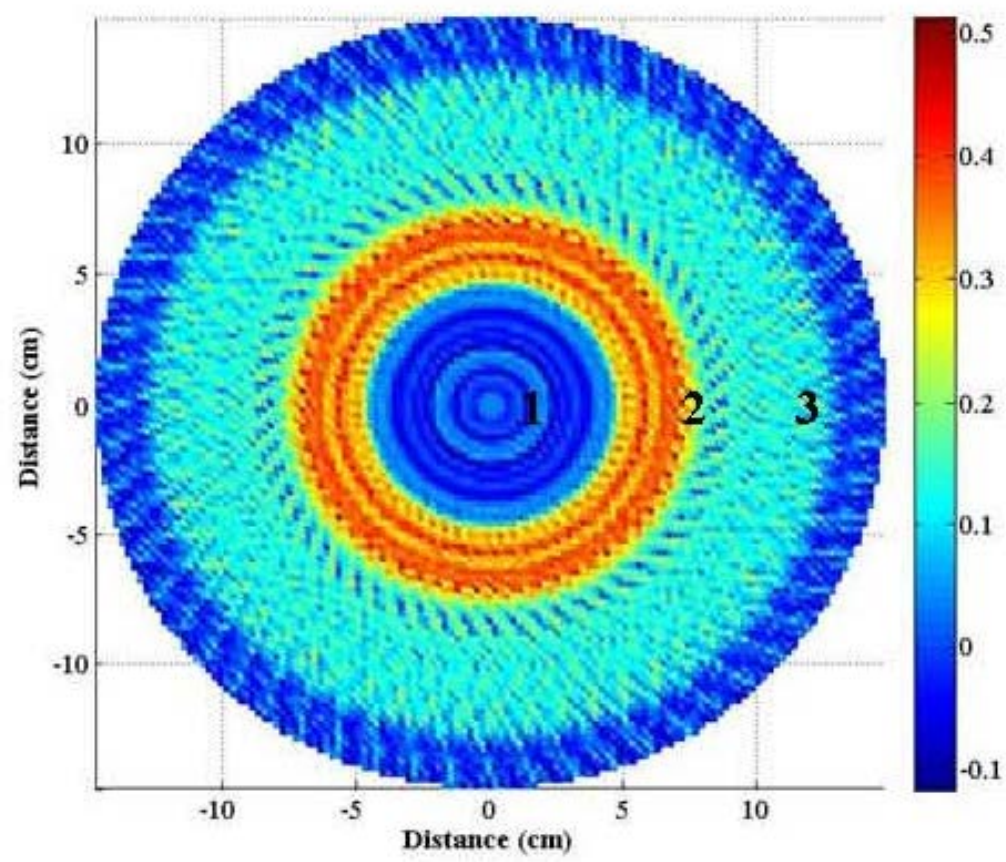

Fig. 12. FBP radial tomograph of Object 1 that plots the attenuation coefficient $\left(\mathrm{cm}^{-1}\right)$ as a function of distance $(\mathbf{c m})$. Red signifies a high attenuation and blue a low attenuation, as shown in the color bar to the right of the image. The three material regions of Object 1 are as follows: (1) void, (2) DU (possibly containing HEU on the interior), and (3) polyethylene.

The results from the passive and active gamma spectroscopy were next used to determine the composition of the material regions visible in the FBP tomograph by matching the attenuation coefficients seen in Fig. 12 with those listed in Table 3. Noise can be observed within the FBP tomograph as a result of the filtering process. For example, between -4 and $+4 \mathrm{~cm}$ in Fig. 12, it appears there are multiple thin rings of some low-attenuating material. However, it is apparent that some of the rings have negative attenuation coefficient values, which is unphysical. Additionally, the noise causes some of the attenuation values to be much higher than they normally would be (most fissile materials and the materials used to shield them have attenuations below $0.3 \mathrm{~cm}^{-1}$ ). The noise problems are statistical and are addressed in Sect. 4.2.3.

When the noise was accounted for, three material regions were delineated: a central void surrounded by DU, and an outermost region composed of polyethylene (Fig. 12). Although HEU may be present, its boundary cannot be determined from the FBP tomograph because its attenuation value is similar to that of DU, and thus at that point in the analysis, only three regions were assumed; a later method determined whether HEU was actually present.

With material regions established, the next step of the process could be undertaken. The boundaries of the three regions labeled in Fig. 12 were approximated by visual inspection of the tomograph and then were entered into a previously written Matlab code, TAKE. The visual inspection values, along with attenuation values for the material regions, were then used as initial guesses to iterate between the measured attenuation length curve in Fig. 11 and a curve that is produced by forward-projecting the guessed object dimensions onto the detectors (Fig. 13). The purpose of running this iterative code is to estimate the dimensions of Object 1 . The resultant TAKE estimates of the dimensions of the three regions are listed in Table 5.

A final result can be drawn from the FBP transmission image when it is combined with the vertical scan image data collected in the previous step. The vertical and radial images lead to the conclusion that Object 1 is cylindrical. 

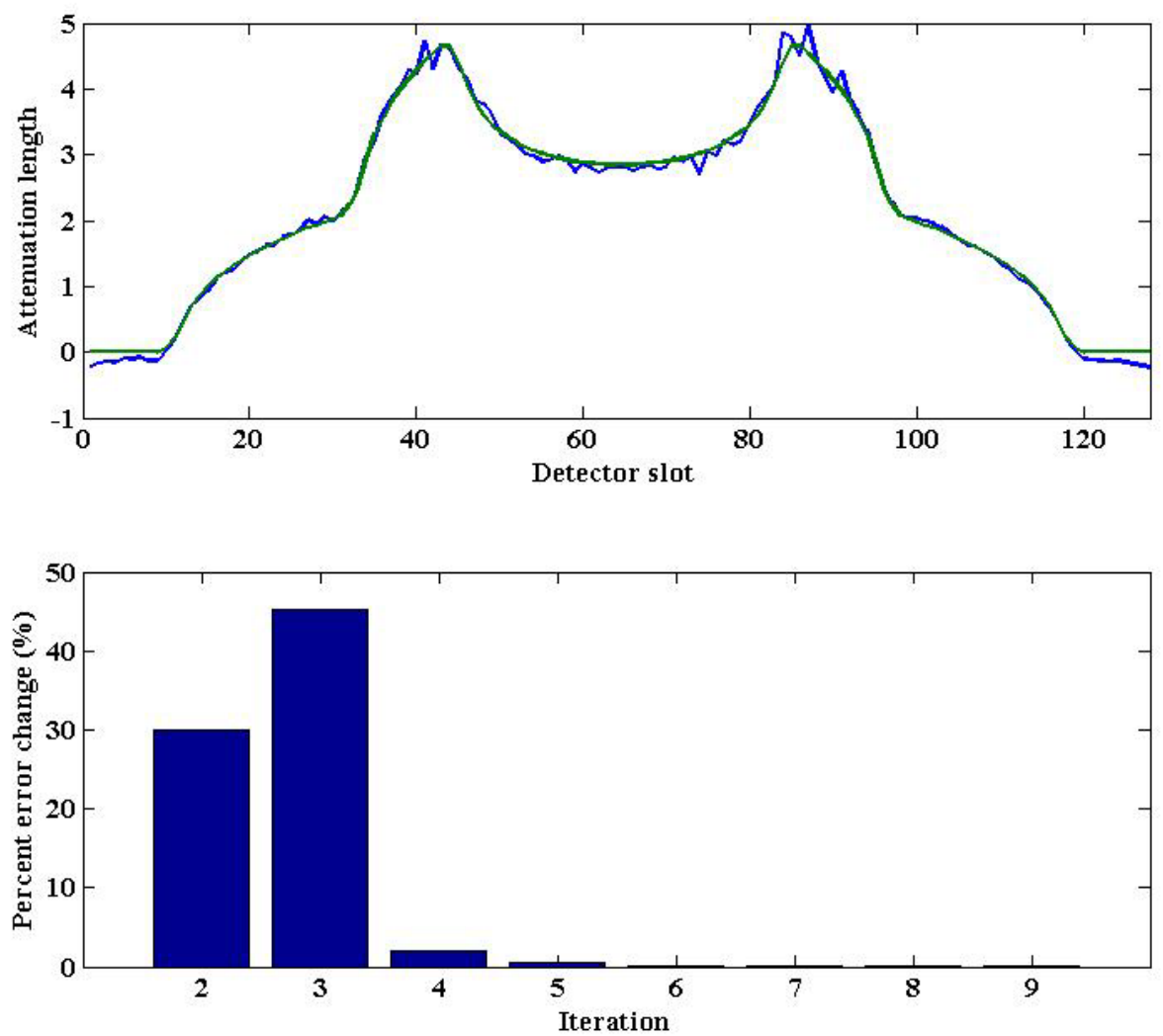

Fig. 13. Top: The TAKE process iterated between the measured attenuation length curve (blue) and one that is produced by forward-projecting the guessed object dimensions onto the detectors (green). Both are for a single rotation.

Bottom: Percentage of error change as a function of number of iterations, showing convergence between the two attenuation length curves.

Table 5. Visual inspection and TAKE radial dimensions and attenuation coefficients for Object 1

\begin{tabular}{ccccc}
\hline Material & \multicolumn{2}{c}{ Visual inspection } & \multicolumn{2}{c}{ TAKE } \\
\cline { 2 - 5 } region & Radius $(\mathbf{c m})$ & $\boldsymbol{\mu}^{\boldsymbol{a}}\left(\times \mathbf{1 0}^{-\mathbf{2}} \mathbf{c m}^{-\mathbf{1}}\right)$ & Radius $(\mathbf{c m})$ & $\boldsymbol{\mu}\left(\times \mathbf{1 0}^{-\mathbf{2}} \mathbf{c m}^{-\mathbf{1}}\right)$ \\
\hline 1 & 4.5 & 0.00 & 4.609 & 0.00 \\
2 & 7.7 & 27.90 & 7.500 & 30.84 \\
3 & 12.5 & 10.98 & 12.352 & $10.89^{b}$ \\
\hline
\end{tabular}

${ }^{a}$ The material-dependent attenuation coefficient for $14.1 \mathrm{MeV}$ source neutrons

${ }^{b}$ This attenuation coefficient is consistent with polyethylene.

\subsubsection{Maximum Likelihood Expectation Maximization Transmission Reconstruction}

MLEM iterative reconstruction was the second method used to form a transmission image of Object 1 from the simulated data. MLEM iterative reconstruction is a different process from FBP imaging in that it begins with an initial guess of 1 for the attenuation coefficient of each pixel in the reconstruction. The attenuation length $(\mu x)$ for each pixel is then projected forward to create a sinogram. The ratio of the current projection to the previous projection is then used as a correction factor that is applied to each pixel [9]. This process was iterated 50 times to generate a final sinogram and then a final transmission image. The initial and final guess sinograms are shown in Fig. 14, with the reconstructed sinogram on the right 
appearing much smoother than the original. Images of the tomographs at iteration 1, 5, and 20, along with the final reconstructed tomograph at iteration 50, can be seen in Fig. 15.
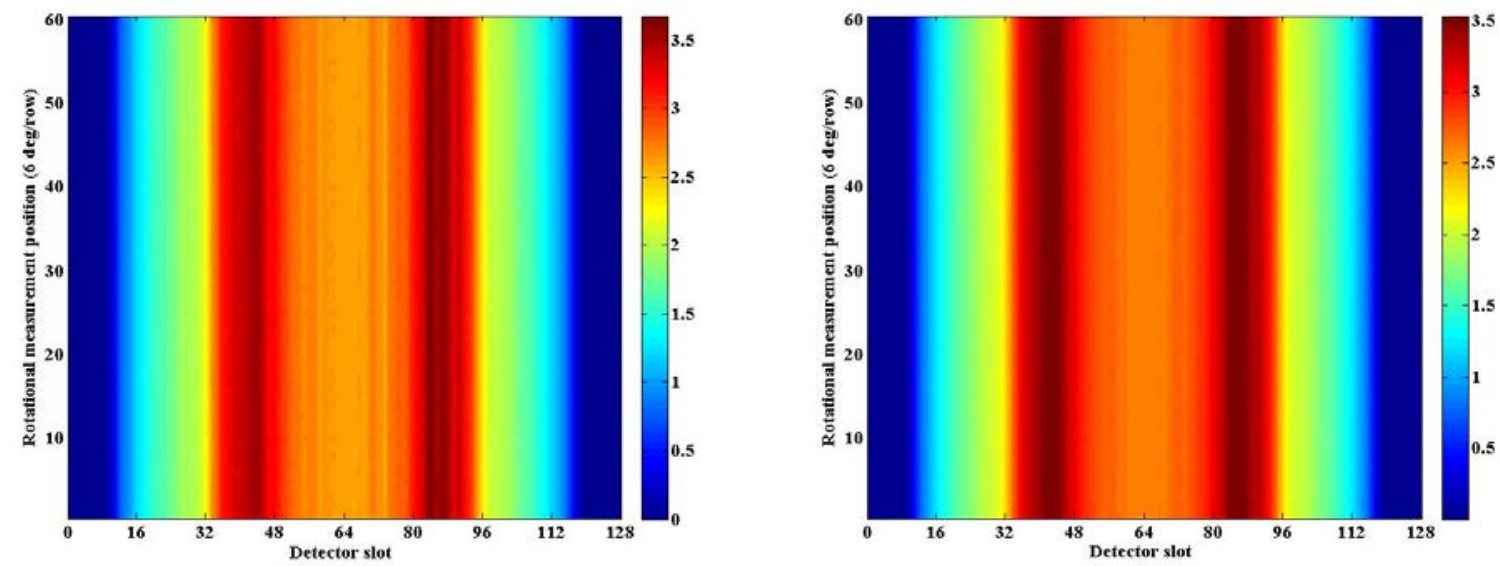

Fig. 14. Initial sinogram (left) and the reconstructed sinogram (right) used to create the MLEM tomograph of Object 1. Areas in red have a high attenuation; areas in blue have a low attenuation.
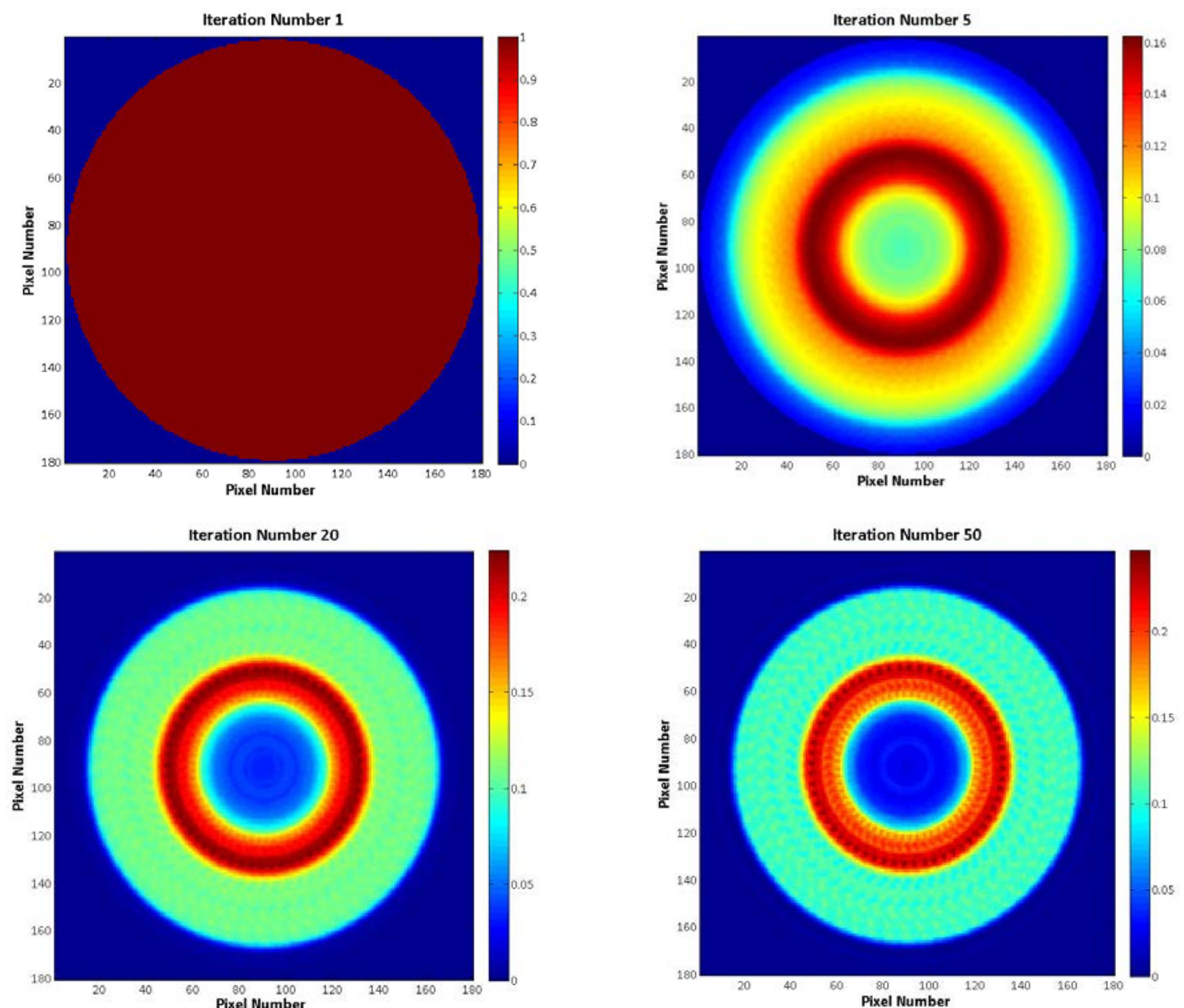

Fig. 15. Reconstructed tomographs at 1, 5, 20, and 50 iterations for Object 1 . Areas in red have a high attenuation, and areas in blue have a low attenuation, as shown in the color bar to the right of the images. Attenuation is shown with units of $\mathrm{cm}^{-1}$. 
The MLEM reconstructed tomograph at iteration 50 has fewer artifacts than the FBP tomograph, and therefore material regions and their attenuation values are much easier to identify (Fig. 16). To determine whether HEU is present and being shielded by DU, it is also necessary to complete MLEM iterative reconstruction of fission sites, a step in the procedure that will be discussed later in this section. However, one cannot rely solely upon the MLEM iterative reconstruction. The FBP method is also necessary to determine the overall dimensions of the object because the FBP method is required to run the TAKE software; therefore, both methods complement each other.
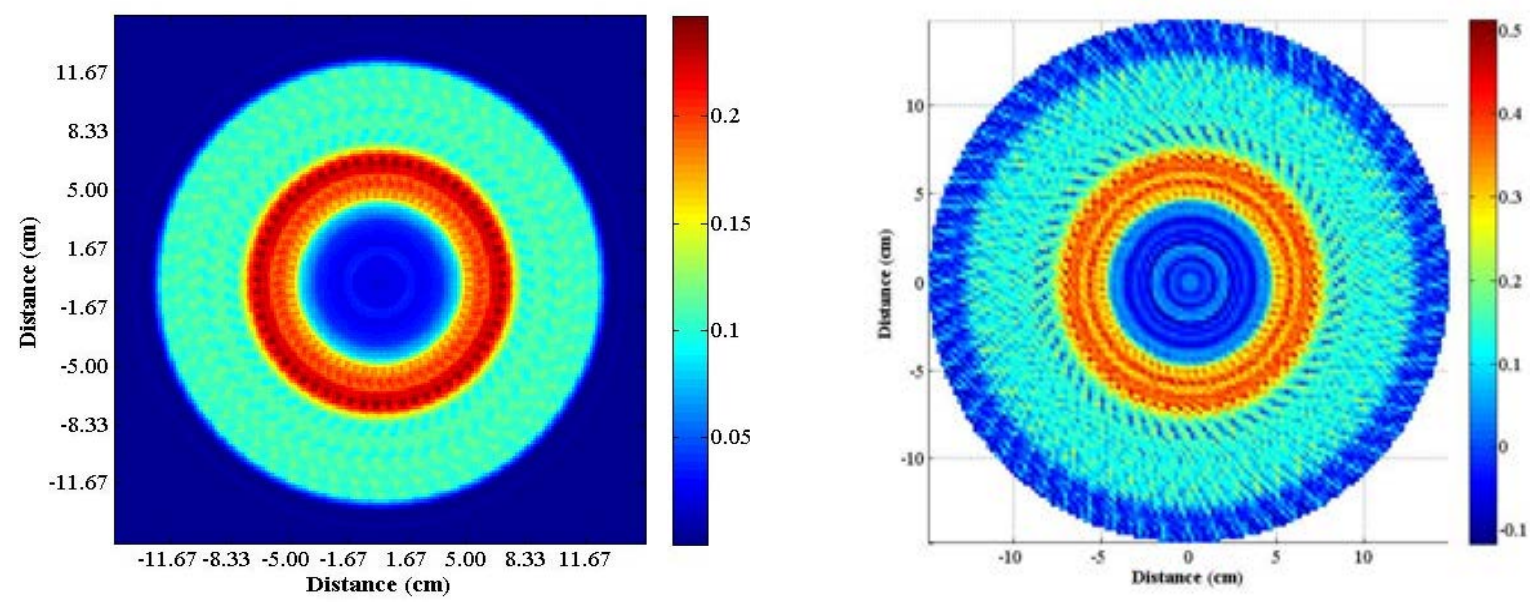

Fig. 16. MLEM tomograph (left) of Object 1 at iteration 50 with adjusted $x$ and $y$ dimensions to show boundary positions. The tomograph from the FBP method (right) is shown here for comparison.

\subsubsection{Fission Mapping Data}

The next step in the procedure was to analyze the fission mapping data. Single and double coincidence counts that had been obtained with the induced fission radiation detectors were correlated with the alpha pixels. In other words, a neutron incident on the induced fission radiation detectors can be correlated with an alpha pixel to determine the origin of the neutron in Object 1 . The data were plotted with Matlab against the corresponding alpha pixel number, thereby reconstructing the locations of fission sites by correlating the fission neutrons measured with the alpha pixel event. These fission mapping plots were useful for determining multiplication because their shapes are dependent solely upon the object's geometry and material composition. The standard deviation, following a Gaussian approximation, was taken to be the square root of the number of simulated counts. A general rule of thumb was that the higher number of counts, and especially the number of doubles counts, the higher the multiplication at that location in the system. The distribution of doubles, plotted in Fig. 17, showed that alpha pixels 5 through 12 define neutrons that impinge on the HEU. 

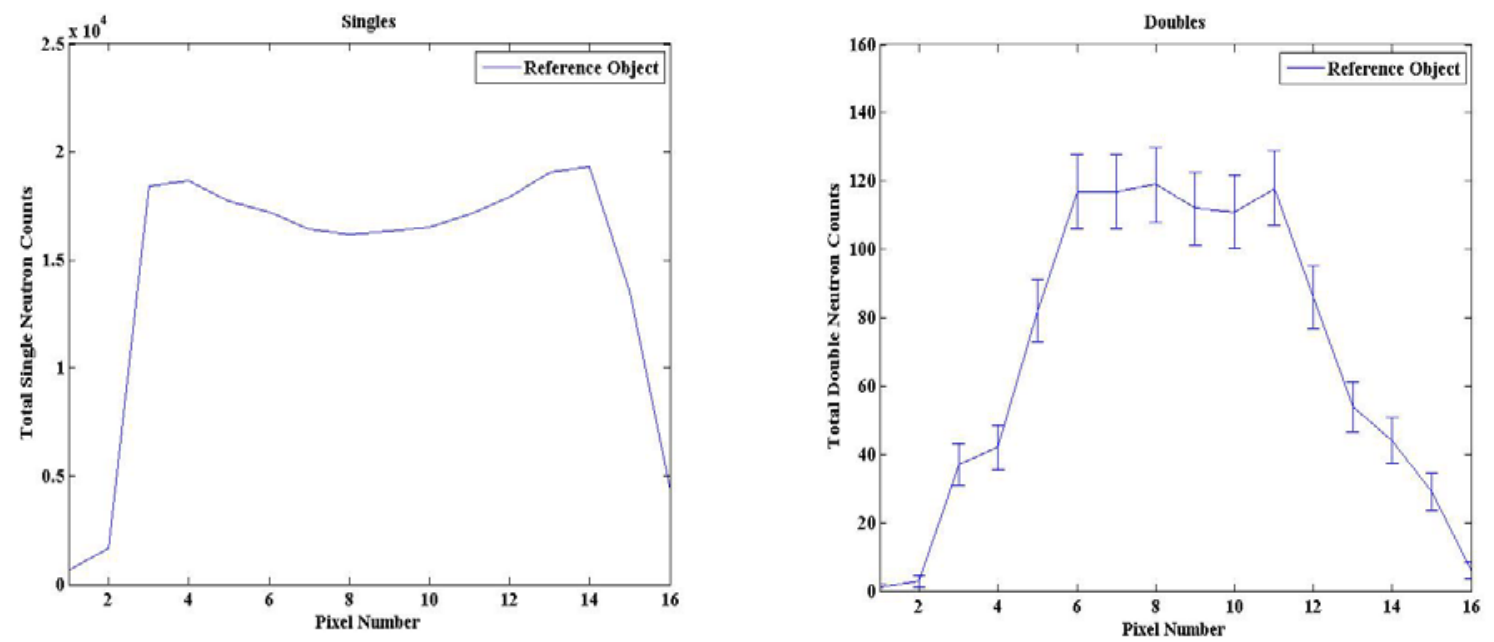

Fig. 17. Fission mapping plots for single (left) and double (right) coincidence counts for the reference data. Pixels 5 through 12 see the most multiplication from the system. Error was taken to be the square root of the counts.

\subsubsection{Multiplicities and Feynman Variances}

As further verification that pixels 5 through 12 were proper choices, the Feynman variance $(Y)$ of the single $\left(y_{1}\right)$ and double $\left(y_{2}\right)$ fission neutron reference data were calculated and plotted against their corresponding alpha pixels $[16,17]$. The multiplication of the reference data that is seen by each pixel can be determined by taking the ratio of doubles to singles counts. Equation (2) was used for the calculation of the Feynman variances:

$$
Y(T)=2\left(\frac{y_{2}}{y_{1}}\right)=2\left(\frac{\left(\begin{array}{l}
2 \\
2
\end{array}\right) b_{2}+\left(\begin{array}{l}
3 \\
2
\end{array}\right) b_{9}}{\left(\begin{array}{l}
1 \\
1
\end{array}\right) b_{1}+\left(\begin{array}{l}
2 \\
1
\end{array}\right) b_{2}+\left(\left(_{1}^{3}\right) b_{3}\right.}\right)=2\left(\frac{b_{2}+3 b_{8}}{b_{1}+2 b_{2}+3 b_{3}}\right)
$$

where $b_{1}$ is the number of singles, $b_{2}$ is the number of doubles, $b_{3}$ is the number of triples, and $T$ is the gate window width. A full derivation of Eq. (2) (which assumes signal-triggered gating) can be found in Appendix D. As can be seen in Fig. 18, pixels 5 through 12 are seeing the multiplication of the system, and thus discarding the others was a proper choice. 


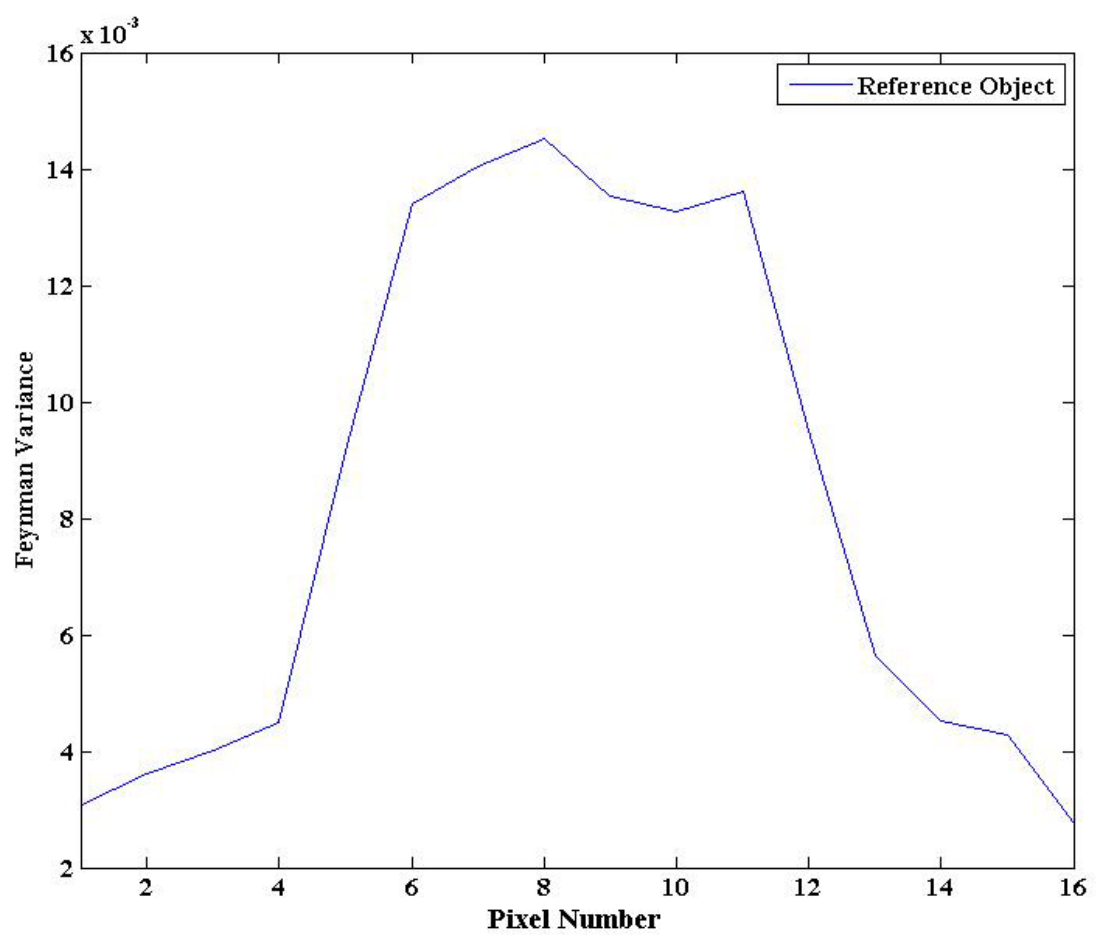

Fig. 18. Feynman variance of the reference data plotted against the alpha pixel number to allow for a visualization of the multiplication seen by each pixel. Here it is obvious that pixels 5 through 12 see the most multiplication, and the outer pixels see little to none.

\subsubsection{Maximum Likelihood Expectation Maximization Fission Reconstruction}

The next step was to utilize the MLEM fission reconstruction methodology. Similar to MLEM transmission reconstruction, MLEM fission reconstruction generates MLEM sinograms and tomographs of the single and double fission neutron sites (Figs. 19 and 20, respectively) and then overlays them in color on a black and white version of the MLEM transmission tomograph (see Fig. 16). The color scale of the MLEM singles and doubles sinograms represents the number of singles (or doubles) detected per alpha particle; the color scale of the singles and doubles images represents the number of singles (or doubles) detected per pixel. Fifty iterations were similarly conducted in this step.

As can be seen in the doubles overlay in Fig. 21, regions of medium and high fission sites (colored yellow and red, respectively) are surrounded by a region of low fission sites (colored white). The images show that initial indications were correct. DU has very few fissions at $0.2 \mathrm{wt} \%{ }^{235} \mathrm{U}$ and would therefore be white in such an image; HEU has many fissions and would be colored red. Thus Object 1 does indeed have a region of HEU surrounded by a dense region of DU. The doubles data provides more insight into the fissile geometry than the singles data, and the singles MLEM fission reconstruction results contain less to provide insight into the region of the fissile geometry due to neutron scatter. 

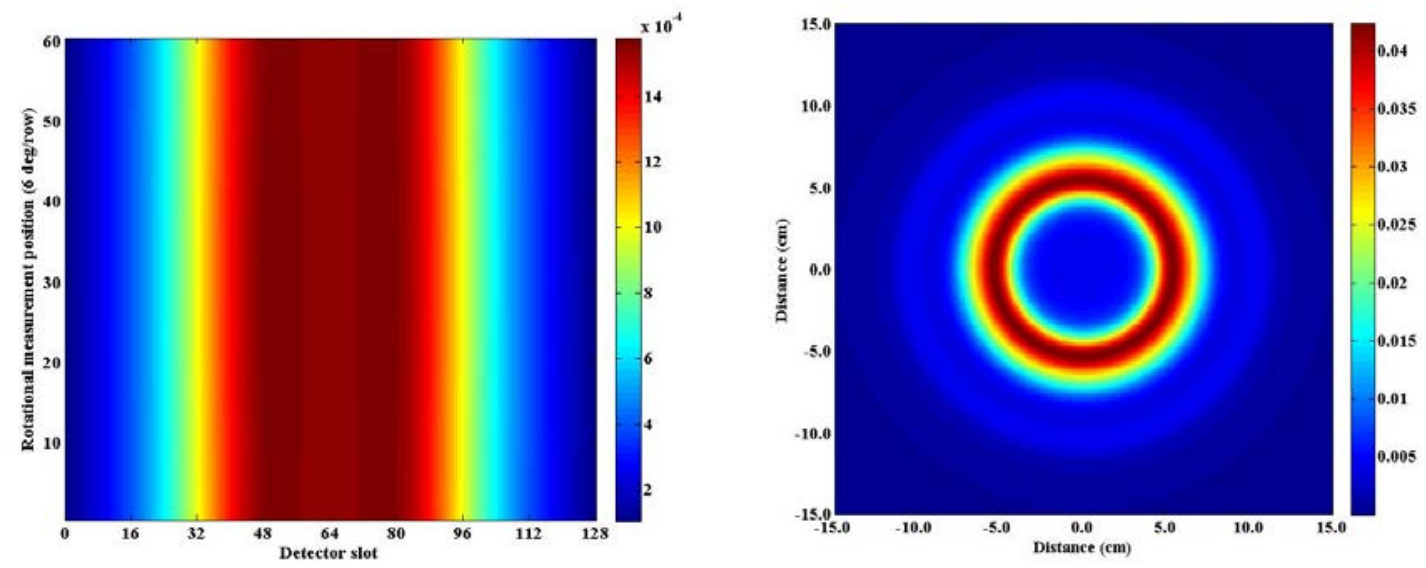

Fig. 19. Singles MLEM reconstruction of the object's sinogram (left) and tomograph (right) after 50 iterations.
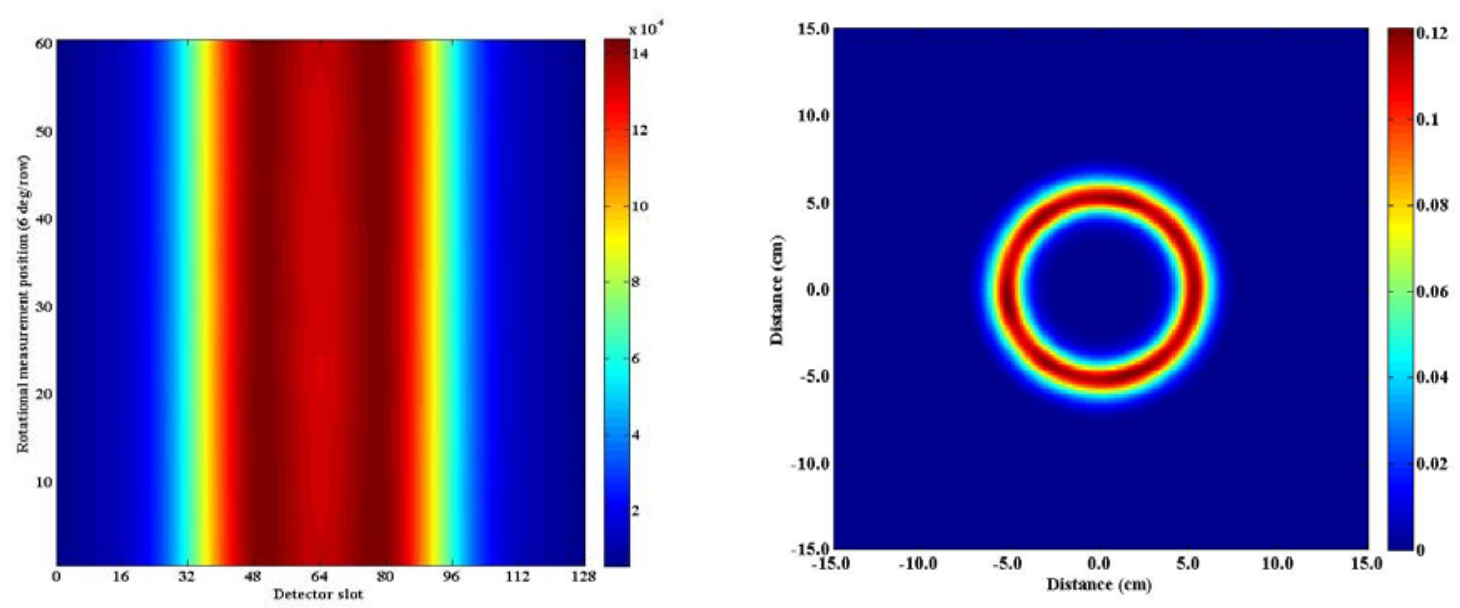

Fig. 20. Doubles MLEM reconstruction of the object's sinogram (left) and tomograph (right) after 50 iterations.
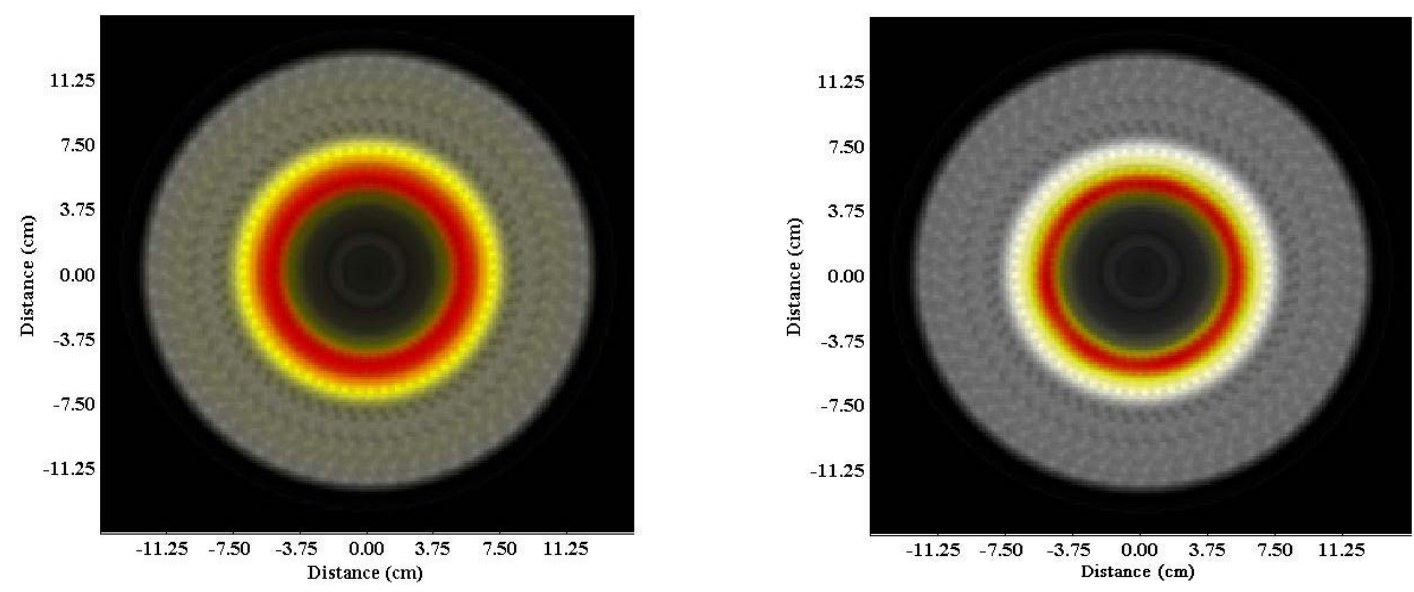

Fig. 21. Overlay of the MLEM tomograph reconstruction for singles (left) and doubles (right) coincidence data. Red indicates high values, yellow mid values, and white low values of fission sites. 
Once it was determined that HEU and DU were both present, the doubles tomograph (see Fig. 20) was used to determine the boundary between the two material regions. The FBP and MLEM transmission images could not be used for that purpose because of the similar attenuation coefficients of DU and HEU, and thus, to determine the outer radius of HEU, the outer radius of the doubles tomograph was examined as follows. A slice of the doubles tomograph was taken at the centerline (i.e., slot 90), which formed a plot of doubles counts against detector slot (Fig. 22). The radius was then approximated to be halfway between the maximum amount of doubles (corresponding to HEU) and near zero doubles (corresponding to DU). Because the HEU formed a cylinder, there were two such locations on the abscissa (54 and 127); converting them into centimeters (dividing them by 6), finding their positive distance from the centerline, and then averaging the two values together yielded an outer radius of HEU of $6.08 \mathrm{~cm}$.

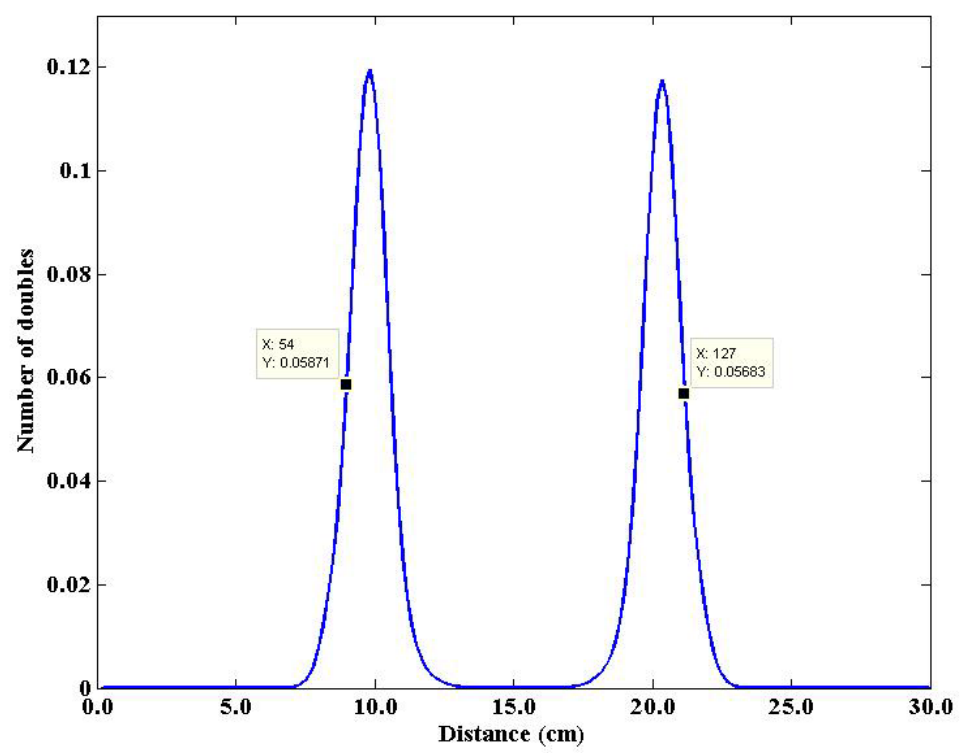

Fig. 22. Centerline slice of the MLEM fission reconstruction doubles tomograph with the two peaks corresponding to the cylindrical region of HEU shielded on the outside by a region of $D U$.

This plot was used to determine the radius of HEU in Object 1.

\subsubsection{Synthesis of Neutron and Photon Data into an Initial Estimate of Object 1}

Synthesis of the gamma and neutron information gathered thus far led to the formation of a guess of the geometry and composition of Object 1 . The analysis thus far was the basis for a MCNP-PoliMi model. The geometry was constructed as follows: a central void inside HEU (enrichment unknown), which was inside regions of DU (0.2 wt \% $\left.{ }^{235} \mathrm{U}\right)$ and polyethylene (Fig. 23).

An interpolation process needed to be conducted to quantify the enrichment of the HEU region (see Sect. 4.3). The process required the creation of four MCNP-PoliMi models of four enrichments of ${ }^{235} \mathrm{U}$ : $40,60,80$, and $93 \mathrm{wt} \%$. The reference time coincidence distribution data could then be compared with the data from the four models to estimate the enrichment of the HEU present in Object 1. 


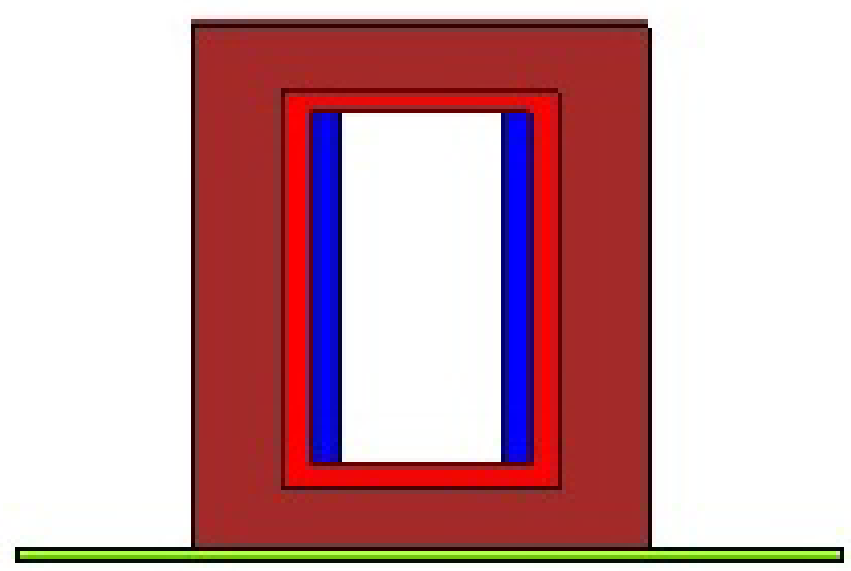

Fig. 23. MCNP-PoliMi geometry showing the material regions: void (white), HEU (blue), DU (red), and polyethylene (dark red). The stainless steel measurement table is also illustrated (green).

\subsection{INTERPOLATION FOR HEU ENRICHMENT DETERMINATION}

\subsubsection{Time Distribution of Coincidences between Detectors and Source Pixels}

Once the four MCNP-PoliMi enrichment models had been created, the first step in the interpolation process for HEU enrichment determination was to examine the time distribution of coincidences between induced fission radiation detectors and each of the associated alpha pixels, known as time of flight (TOF) distributions. The TOF plots were generated using the singles and doubles from the induced fission radiation detectors. The plots consisted of the sum of the number of all coincidences (over all rotations) vs the time they were detected, with one graph for each of the sixteen detector pixels. For each pixel, the four models of varying enrichment and the reference data were plotted, yielding five curves per figure. An example plot can be seen in Fig. 24, which shows the TOF plot for pixel 9. 


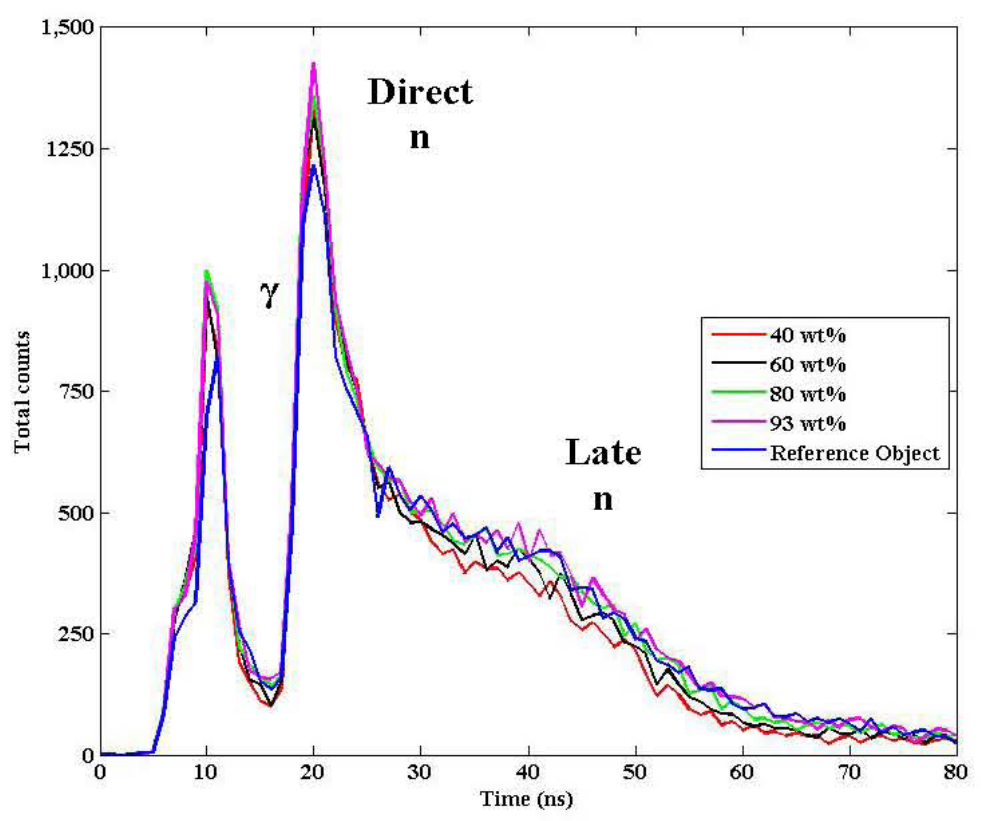

Fig. 24. Pixel 9 TOF plot of the total singles counts vs time for the four MCNP-PoliMi models (red, black, green, and pink) and for the reference data (blue). Gamma $(\gamma)$, and direct and late neutron (n) peaks are labeled.

Several significant facts can be obtained from the TOF plots. Photons, produced by inelastic scatter within the object and traveling at the speed of light, arrive much more quickly than direct neutrons and are detected first (gammas arrive in about 7 to $10 \mathrm{~ns}$ ); thus, two distinct peaks are visible in any TOF plot (Fig. 24). "Late" neutrons often cause a slight rise in counts after the sharp initial neutron peak. Its position in time is the result of the time it takes for the source neutrons to reach the HEU and on the TOF of induced fission radiation to reach the detectors. Its magnitude is indicative of fission multiplication. For this reason, that region is an indicator of enrichment level, everything else being the same; the higher the counts in this region, the greater the enrichment or fissile mass that is present. It can be seen in the TOF plot in Fig. 24 that the "late" neutrons are detected from approximately 35 to 80 ns.

\subsubsection{Creation of Enrichment Curves for Interpolation for Enrichment Determination}

With TOF plots thus generated for every pixel, enrichment curves could be created for all sixteen pixels, as well. However, as was established earlier with the fission mapping studies, only alpha pixels 5 through 12 were associated with the neutrons incident on the HEU region (Fig. 17). Therefore, they were the only pixels used for the analysis. The reasoning for this is similar to the logic for integrating between 35 and 80 ns and is derived from the fact that the effects (i.e., neutron scatter instead of fission) of other materials present in Object 1 needed to be limited.

Thus, eight enrichment curves were generated as follows. Each TOF plot had five curves: four for the four MCNP-PoliMi models and one for the reference data (Fig. 24). All five were integrated between 35 and $80 \mathrm{~ns}$ to obtain the induced fission radiation fission counts resulting from the HEU. The four integrated counts resulting from the four MCNP-PoliMi models of varying enrichment were then plotted against their enrichment in weight percent to produce an enrichment curve for interpolation for enrichment determination for pixels 5 through 12. An example curve can be seen in Fig. 25. The uncertainty was taken to be the square root of the counts, and enrichment curves were fitted with a second-order polynomial, which is detailed in Appendix E. 


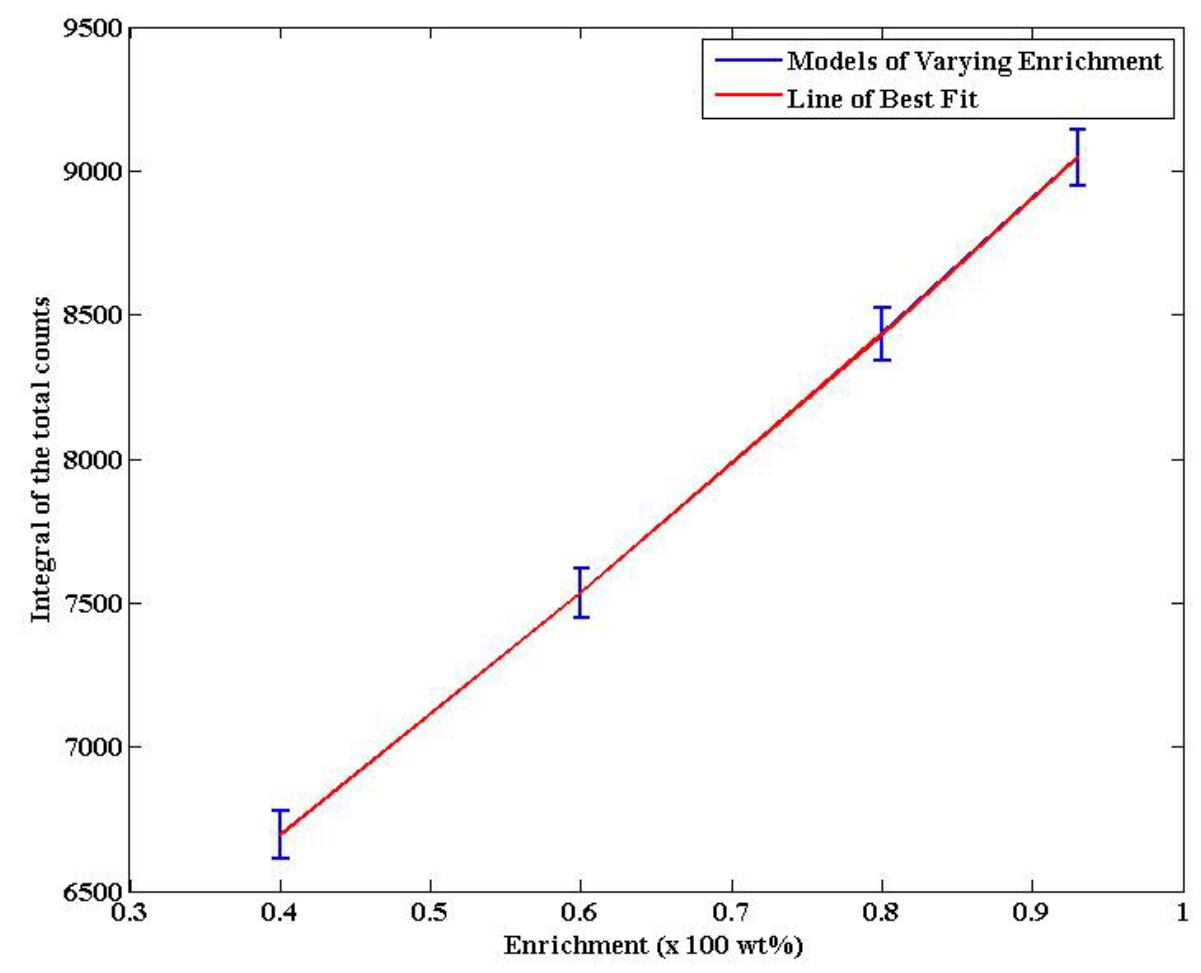

Fig. 25. Pixel 9 enrichment curve generated from the integral of the four MCNP-PoliMi models' TOF plots from 35 to 80 ns. Error was taken to be the square root of the counts.

The next step in determining the enrichment of Object 1 was to use the integral of the reference counts to interpolate from the eight enrichment curves. With the integral of the reference data (and therefore a value on the $y$-axis of the enrichment curve) and the equation of the line of best fit both known, the quadratic formula was used to determine the enrichment. This was equivalent to drawing a horizontal line at a height equal to the integral of the total reference counts from the $y$-axis to the enrichment curve, and then drawing a vertical line from the intersection point down to the $x$-axis to determine the enrichment. This is illustrated for pixel 9 in Fig. 26, which had an integrated reference counts value of 8783 counts and resulted in an enrichment of $87.5 \mathrm{wt} \%{ }^{235} \mathrm{U}$. 


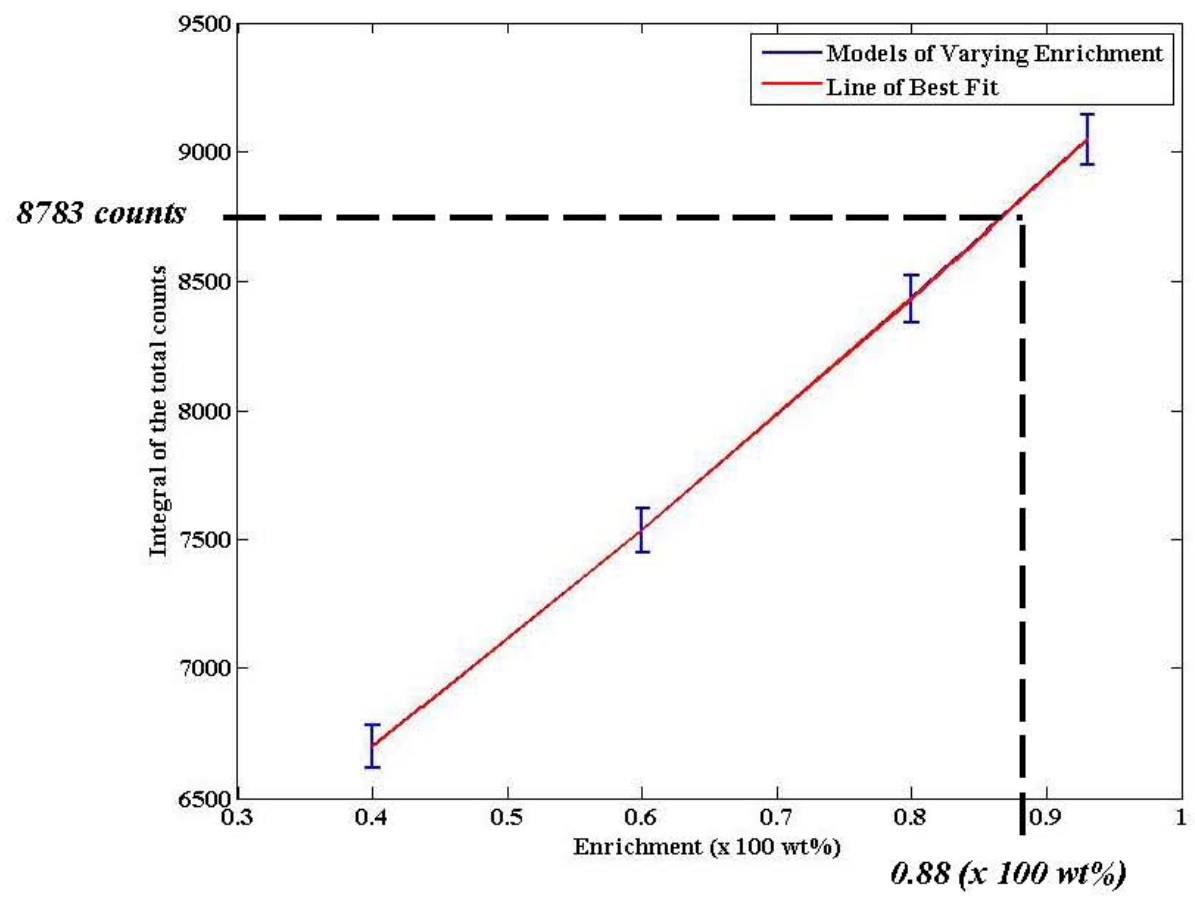

Fig. 26. Illustration of the interpolation method equivalent to the quadratic formula used to determine the enrichment of the HEU region of Object 1 . The plot and numbers provided are for Pixel 9. Error was taken to be the square root of the counts.

Upper and lower bounds of enrichment were similarly found for pixels 5 through 12 by using the respective upper and lower endpoints of the error bars, which corresponded to $1 \sigma$. Enrichment values for the eight pixels in question, as well as their average values, can be seen in Table 6. It was therefore determined that the average enrichment (averaged over the eight pixels in question) of the HEU present in Object 1 was 84.7 wt $\%{ }^{235} \mathrm{U}$, with $1 \sigma(68.3 \%)$ probability of being between 82.8 and $86.6 \mathrm{wt} \%{ }^{235} \mathrm{U}$.

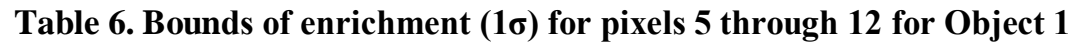

\begin{tabular}{cccc}
\hline Pixel number & $\boldsymbol{E}_{\text {low }}$ (wt \%) & $\boldsymbol{E}$ (wt \%) & $\boldsymbol{E}_{\text {high }}$ (wt \%) \\
\hline 5 & 78.6 & 80.9 & 83.1 \\
6 & 84.4 & 86.3 & 88.3 \\
7 & 84.1 & 85.8 & 87.4 \\
8 & 85.1 & 86.7 & 88.3 \\
9 & 85.5 & 87.5 & 89.5 \\
10 & 85.4 & 87.2 & 89.0 \\
11 & 81.5 & 83.1 & 84.7 \\
12 & 77.9 & 80.3 & 82.6 \\
Average & 82.8 & 84.7 & 86.6 \\
\hline
\end{tabular}





\section{DESCRIPTION OF THE UNKNOWN OBJECT}

After the completion of the examination of the simulated neutron and gamma data, the final estimate of Object 1 was that it was a cylindrical object with the dimensions and material regions listed in Table 7. DU and HEU were 0.2 wt \% and 84.7 wt $\%{ }^{235} \mathrm{U}$, respectively. The true geometry of Object 1 was later revealed (also included in Table 7). The mass of ${ }^{235} \mathrm{U}$ was also calculated for the HEU and DU regions for the final estimate and actual geometry (Table 8).

Table 7. Final estimate and actual geometry of Object 1

\begin{tabular}{ccccc}
\hline Material region & \multicolumn{2}{c}{ Final estimate } & \multicolumn{2}{c}{ Actual geometry } \\
\hline & Radius (cm) & Height $\mathbf{( c m )}$ & Radius (cm) & Height (cm) \\
\hline Void & 4.609 & 17 & 4.445 & 15.24 \\
HEU & 6.080 & 17 & 6.350 & 15.24 \\
DU & 7.500 & 19 & 7.620 & 17.80 \\
Polyethylene & 12.352 & 25 & 12.700 & 25.40 \\
\hline
\end{tabular}

Table 8. Mass of ${ }^{235} \mathrm{U}$ in the final estimate and actual geometry of Object 1

\begin{tabular}{|c|c|c|c|}
\hline \multirow{2}{*}{ Fissile material } & \multicolumn{2}{|c|}{${ }^{235} \mathrm{U}$ mass } & \multirow{2}{*}{$\begin{array}{c}\text { Percent error } \\
\mathbf{( \% )}\end{array}$} \\
\hline & Final estimate & Actual geometry & \\
\hline HEU & $13.43 \mathrm{~kg}$ & $14.89 \mathrm{~kg}$ & -9.7 \\
\hline DU & $52.29 \mathrm{~g}$ & $49.76 \mathrm{~g}$ & -5.1 \\
\hline
\end{tabular}

Uncertainty analysis of radial and height dimensions, as well as of the volume of each material region, can be seen in Table 9. Most of the error results from the heights of the components of Object 1 , which were derived from the vertical scan. This is because the vertical scan image is less clear than the radial scan. Enrichment estimates (and therefore ${ }^{235} \mathrm{U}$ mass) would have also been more accurate with more accurate radial and height dimensions; enrichment would have decreased to compensate from 84.7 wt \% toward the actual enrichment of $80.0 \mathrm{wt} \%{ }^{235} \mathrm{U}$ due to multiplication [18]. Additional data that could increase the speed and accuracy of geometry analysis are detailed in Chap. 6.

Table 9. Percent difference between final estimate and actual geometry of Object 1

\begin{tabular}{ccc}
\hline Material region & Radial percent error (\%) & Height percent error (\%) \\
\hline Void & 3.69 & 12 \\
HEU & -4.25 & 12 \\
DU & -1.58 & 7 \\
Polyethylene & -2.74 & -2 \\
\hline
\end{tabular}





\section{ANALYSIS OF THE ITERATIVE METHODOLOGY}

\subsection{FURTHER ANALYSIS OF AUTO- AND CROSS-CORRELATIONS}

A similar analysis of Object 1 should be performed using the auto- and cross-correlation functions between detectors to determine the enrichment. These functions yield plots similar in shape and meaning to TOF plots in that they have an initial gamma peak followed by a neutron peak that comprises direct and late neutrons. However, auto- and cross-correlations plot the time behavior of coincident neutron doubles, whereas TOF plots are for neutron singles. As a result, auto- and cross-correlations may be a better measure of multiplication than TOF plots and therefore may be better at estimating through interpolation the enrichment of the fissile material present in the unknown object.

\subsection{FISSION MAPPING ALONG THE VERTICAL AXIS}

Another technique that should be considered for future use with NMIS measurements and simulations of measurements is fission mapping along the vertical axis. It would alleviate the problem outlined previously that has been encountered with transmission imaging: DU and HEU appear the same in a transmission image because they have similar attenuation coefficients. By mapping fission sites along the vertical axis of an unknown object, any layers of DU adjacent to the top and bottom HEU could be more concretely determined. In the current study, for example, it would have shown, rather than being assumed, that a layer of DU was on the top and bottom of the HEU region in Object 1.

\subsection{FURTHER ANALYSIS OF THE METHOD TO DETERMINE THE RADIUS OF THE HEU REGION}

When determining the HEU-DU boundary from the slice of the doubles MLEM fission reconstruction, it had been assumed that a valid approximation was the halfway point between the maximum amount of doubles (corresponding to HEU) and near zero doubles (corresponding to DU). Further attention should be given to this methodology to optimize it, if possible, and to increase accuracy with respect to dimensions and enrichment. 



\section{SUMMARY AND CONCLUSIONS}

Simulated transmission neutron imaging data, induced fission radiation detector data (MCNPPoliMi), and passive and active gamma spectroscopy data (MCNPX version 2.6.0) were provided for analysis to determine the geometry and material composition of an unknown object (“Object 1"). Using a previously determined procedure, the passive gamma spectrum was first analyzed for the presence of fissile material peaks. The peaks were then used to estimate enrichment and were compared with the results of GADRAS, a multiple-regression iterative analysis code. The active gamma spectrum was then inspected to determine the presence of nonfissile material in Object 1. The results of those two steps were next combined to determine that DU and polyethylene were also possible materials present in Object 1.

Next, neutron transmission imaging data were studied. An FBP vertical scan of the object was examined to determine the heights of material regions at which to obtain a radial neutron transmission image. Two radial neutron transmission images of Object 1 were then formed using algorithms that implement the FBP and MLEM iterative reconstruction transmission imaging methods. The images were studied to determine the number of material regions and their material attenuation coefficients (and thus material type, cross-referenced with the list resulting from the gamma spectra). The radii of the material regions were then found using TAKE, an iterative fitting algorithm that determined the dimensions and attenuation coefficients. It was also then determined that Object 1 had a cylindrical geometry. To ascertain whether HEU was present and being shielded by an outer region of DU, MLEM fission site reconstruction was employed. Since most of the fissions were occurring inside the DU metal, the reconstruction indicated that HEU metal was on the inside.

At that point, an estimate of the geometry of Object 1 was formed: a central void surrounded by HEU of unknown enrichment, nested inside DU $\left(0.2 \mathrm{wt} \%{ }^{235} \mathrm{U}\right)$, with an outer shell of polyethylene. That geometry was entered into a MCNP-PoliMi model that had four different enrichments of HEU to compare the calculated time distribution of coincidences as a function of enrichment between the induced fission radiation detectors and the alpha pixels to the reference data. Fission mapping also determined which pixels induced fission in the HEU (pixels 5 through 12). The Feynman variance as a function of pixel number was also calculated for each pixel to corroborate the assumption that pixels 5 through 12 were associated with neutrons that were incident on the HEU region of Object 1.

After corresponding TOF plots between 35 and 80 ns (the time at which induced fission neutrons reach the fission radiation detectors) were integrated and after the integrals of the counts were plotted against the enrichment, the integral of the counts from the reference data was used to interpolate and find a pixel-specific enrichment of the HEU in Object 1 . The pixel-specific enrichments were then averaged to yield an overall estimate of $84.7 \mathrm{wt} \%{ }^{235} \mathrm{U}$. Statistical uncertainty (taken to be the square root of the counts) was used to find upper (86.6 wt \%) and lower (82.8 wt \%) bounds of $1 \sigma$ on the estimate. The actual enrichment was $80 \mathrm{wt} \%$, so the estimate was within $2.5 \sigma$ of the true value. The estimate could be improved by longer count times, which would reduce error.

Error between the estimated dimensions and the actual geometry varied. Radially, relative error varied from $1.58 \%$ to $-4.25 \%$, with the greatest error in the HEU region; heightwise, relative error ranged from $-2 \%$ to $12 \%$. Relative error in the estimate of the enrichment of $5.9 \mathrm{wt} \%$. Most of the disparity resulted from systematic error introduced by the algorithm that conducted the vertical neutron transmission image reconstruction.

In summary, it is possible to quantitatively determine the materials present and their configuration by iterative analysis using passive and active gamma spectroscopy, FBP and MLEM neutron transmission tomographs (including radial and vertical scans), neutron coincidence and multiplicity counting, and fission mapping data. The unknown object presented here represents a case that is particularly challenging because the fissile material was surrounded by DU. Even so, the region that contains the HEU was identified, and its enrichment was determined within $2.5 \sigma$. Therefore, the level of accuracy achieved in the study may be adequate for possible use in future treaty verifications, but the specifics would depend on the agreement between treaty partners. With future work on more complicated material configurations, 
auto- and cross-correlations, and fission mapping along the vertical axis, it may be possible to reduce uncertainties further and apply this technology to treaty verification. 


\section{REFERENCES}

J. T. Mihalczo, J. A. Mullens, J. K. Mattingly, and T. E. Valentine, "Physical description of nuclear materials identification system (NMIS) signatures," Nuclear Instruments and Methods in Physics Research A 450, 531-555 (2000).

J. T. Mihalczo, J. K. Mattingly, J. S. Neal, and J. A. Mullens, "NMIS plus gamma spectroscopy for attributes of HEU, PU, and HE detection," Nuclear Instruments and Methods in Physics Research B 213, 378-384

(2004).

P. A. Hausladen, P. R. Bingham, J. S. Neal, J. A. Mullens, and J. T. Mihalczo, "Portable fast-neutron radiography with the nuclear materials identification system for fissile material transfers," Nuclear Instruments and Methods in Physics Research B 261, 387-390 (2007). MCNP-PoliMi, http://www-rsicc.ornl.gov/codes/ccc/ccc7/ccc-718.html. MCNPX, http://mcnpx.lanl.gov.

J. Mihalczo and J. Mullens, Nuclear Material Identification System with Imaging and Gamma-Ray Spectrometry for Plutonium, Highly Enriched Uranium, High Explosives, and Other Materials, ORNL/TM2012/22, Oak Ridge National Laboratory, Oak Ridge, Tenn. (February 2012).

J. Mullens, S. McConchie, P. Hausladen, J. Mihalczo, B. Grogan, and E. Sword, "Neutron radiography and fission mapping measurements of nuclear materials with varying composition and shielding," 52nd

Annual INMM Meeting Conference Proceedings (July 2011).

G. F. Knoll, Radiation Detection and Measurement, Third ed., 26-27 (2000).

B. R. Grogan, J. T. Mihalczo, S. M. McConchie, and J. A. Mullens, "Identification of shielding material configurations using NMIS imaging," 52nd Annual INMM Meeting Conference Proceedings (July 17, 2011).

K. Peña, S. McConchie, J. Crye, and J. Mihalczo, "Active interrogation observables for enrichment determination of DU shielded HEU metal assemblies with limited geometrical information," 52nd Annual INMM Meeting Conference Proceedings (July 2011).

B. R. Grogan and J. T. Mihalczo, "Simulating NMIS imaging and fission-mapping measurements," 52nd Annual INMM Meeting Conference Proceedings (July 2011).

B. R. Grogan, S. M. McConchie, J. T. Mihalczo, and J. A. Mullens, "Alpha detector pixelation effects on NMIS imaging," 49th Annual Meeting of the Institute of Nuclear Materials Management Conference Proceedings (November 2008).

G. W. Phillips, D. J. Nagel, and T. Coffey, A Primer on the Detection of Nuclear and Radiological Weapons, Center for Technology and National Security Policy, National Defense University (2005).

D. Reilly, N. Ensslin, H. Smith, Jr., and S. Kreiner, Passive Nondestructive Assay of Nuclear Materials, US Nuclear Regulatory Commission (1991).

GADRAS, http://www-rsicc.ornl.gov/codes/psr/psr5/psr-560.html.

R. P. Feynman, D. de Hoffmann, and R. Serber, Dispersion of the Neutron Emission in U235 Fission, Report for Los Alamos National Laboratory (1956).

S. Fredriksen, "Spatial distribution of induced fission from the pixilated alpha detector in a D-T generator," Master's thesis, University of Tennessee, Knoxville, 2009.

J. Duderstadt and L. Hamilton, Nuclear Reactor Analysis, Wiley, 84 (1976). 

APPENDIX A. PROCESS FOR THE ANALYSIS OF NMIS TYPE NEUTRON IMAGING AND GAMMA SPECTROSCOPY 



\section{APPENDIX A. PROCESS FOR THE ANALYSIS OF NMIS TYPE NEUTRON IMAGING AND GAMMA SPECTROSCOPY}

The following procedure for analyzing an NMIS-type neutron imaging and gamma spectroscopy and data flowchart (Fig. A.1) is quoted from J. Mihalczo and J. Mullens, Nuclear Material Identification System with Imaging and Gamma-Ray Spectrometry for Plutonium, Highly Enriched Uranium, High Explosives, and Other Materials, Global Nuclear Security Technology Division Report for Oak Ridge National Laboratory (February 2012) [6].

1. Perform passive gamma-ray spectrometry measurements with no object to determine background.

2. Perform passive NMIS time correlation measurements with no object to determine background and determine time coincidence distributions and multiplicities.

3. Perform baseline I0 measurement with no object with the subsampling that will be used later for the vertical radiographic image scan and the tomographic slice image. (I0 is the neutron flux counted when no target is present, that is, a calibration of the efficiency of the transmission detection system.)

4. Perform gamma-ray spectrometry measurements with the sources turned on to obtain the active background from the nearby materials.

5. Locate the target object appropriately and measure its location.

6. Perform passive gamma-ray spectrometry with object.

7. Perform passive time correlation measurements to see if plutonium or HEU is present.

8. Evaluate the passive data to make preliminary conclusions including running a GADRAS-like code.

9. Perform a shadowgraph (radiograph) imaging scan to determine overall shape of the object and find location of interest for more detailed imaging.

10. Perform detailed tomographic slice image measurements at heights of interest to determine the internal configuration. Measure at least two turntable rotary positions if possible.

11. Perform time-tagged gamma spectrometry at the same time as step 10 at locations of interest to assist in the identification of materials.

12. Reconstruct transmission data using MLEM algorithm. Use resulting images to create the initial guess for dimensions and attenuation coefficients of individual parts inside of the object.

13. Run TAKE to determine dimensions and attenuation coefficients of individual parts using information obtained in step 10.

14. Reconstruct fission site images from multiplicity singles and doubles data using MLEM algorithm.

15. Overlay singles and doubles reconstructions onto transmission reconstructions to identify which internal parts are composed of fissile material.

16. Look at the neutron scattering time-of-flight data for each pixel to identify light materials. 
17. Look at passive and active gamma-ray spectra to determine the presence of and the relative amounts of isotopes of interest in the object. Subtraction of the passive data from the active should give the non-elastic gamma production to identify explosives and other materials.

18. Come up with a simple 1-D model using the results from previous steps and fit the model parameters to the passive gamma spectra using GADRAS.

19. Develop a MCNP model of the system based on the previous steps.

20. Run MCNP simulations of measured quantities (possibly including transmission, fission mapping, neutron scattering, and active gamma spectrometry).

21. Generate passive gamma spectrum using the GADRAS 1-D model.

22. Compare results of steps 20-21 with measurements and generate a goodness-of-fit value.

23. Check on the goodness-of-fit result for convergence. If converged, then end; otherwise, modify model dimensions and materials and return to step 20.

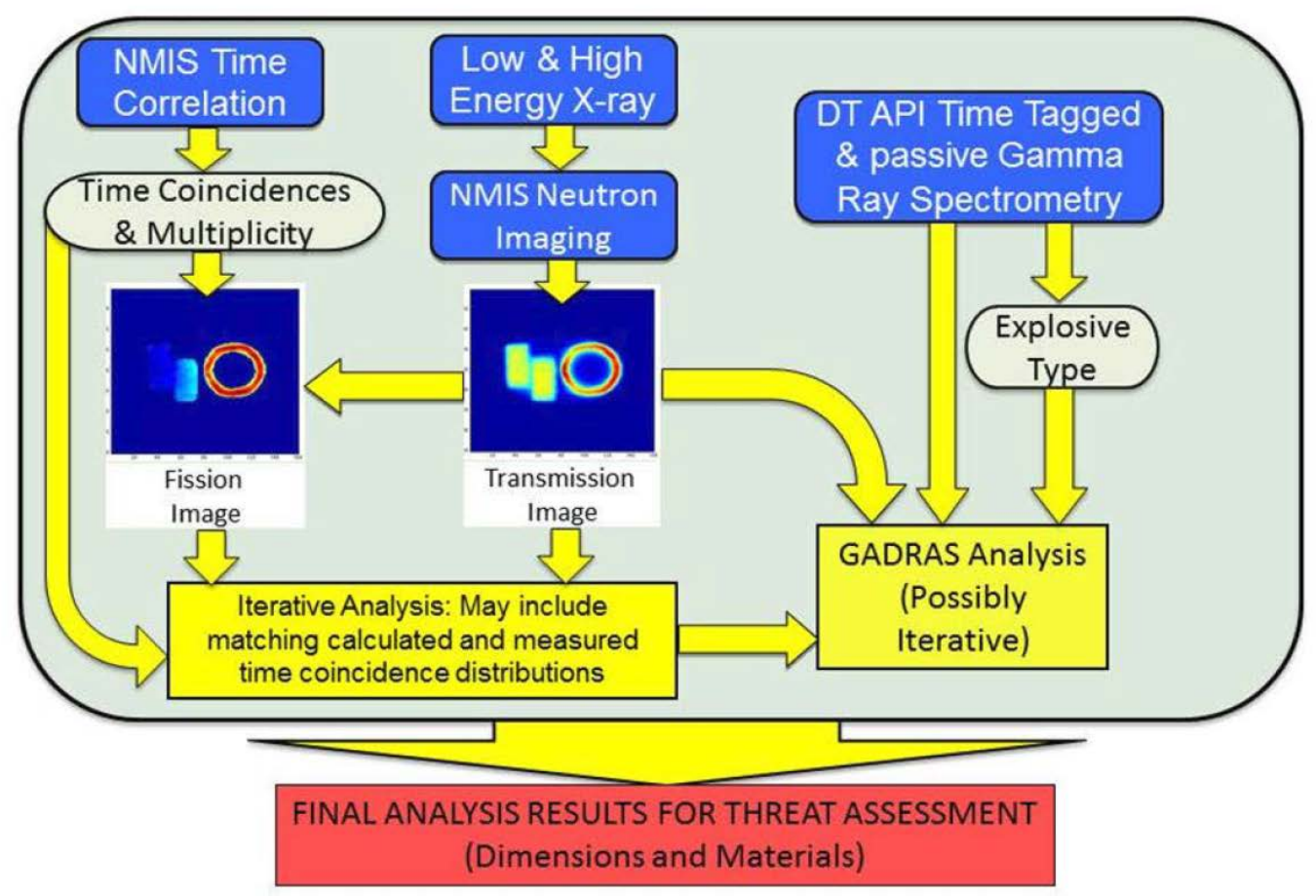

Fig. A.1. General flowchart depicting the steps necessary for analyzing NMIS type neutron imaging and gamma spectroscopy data. Source: J. Mihalczo and J. Mullens, Nuclear Material Identification System with Imaging and Gamma-Ray Spectrometry for Plutonium, Highly Enriched Uranium, High Explosives, and Other Materials, Global Nuclear Security Technology Division Report for Oak Ridge National Laboratory (February 2012) [6]. 
APPENDIX B. ESTIMATION OF URANIUM ENRICHMENT FROM 186-1001 KEV GAMMA LINES 



\section{APPENDIX B. ESTIMATION OF URANIUM ENRICHMENT FROM 186-1001 KEV GAMMA LINES}

The equations below use the following variables, with their values listed in Table B.1:

- $t$ (s) is the live time of the passive gamma spectroscopy measurement

- $A_{186}, A_{1001}$ are the net counts under the $186 \mathrm{keV}$ and $1001 \mathrm{keV}$ peaks, respectively

- $\quad T_{\frac{1}{2}, 235}, T_{\frac{1}{2}, 238}(\mathrm{~s})$ are the half-lives of ${ }^{235} \mathrm{U}$ and ${ }^{238} \mathrm{U}$, respectively

- $\quad Y_{186}, Y_{1001}$ are the fraction of decays that produce the $186 \mathrm{keV}$ and $1001 \mathrm{keV}$ peaks, respectively

- $\quad N_{235}, N_{238}$ are the number of ${ }^{235} \mathrm{U}$ and ${ }^{238} \mathrm{U}$ atoms

- $\quad E$ (wt \%) is the estimate of the ${ }^{235} \mathrm{U}$ enrichment

Table B.1. 186 and $1001 \mathrm{keV}$ net peak areas, fraction of decays, and half-lives for ${ }^{235} U$ and ${ }^{238} U$ used to estimate enrichment of Object 1

\begin{tabular}{|c|c|c|c|c|c|c|c|}
\hline$A_{186}$ & $A_{1001}$ & $Y_{186}(\%)$ & $Y_{1001}(\%)$ & $T_{\frac{1}{2} 235}(\mathrm{~s})$ & $T_{\frac{1}{z} 238}(\mathrm{~s})$ & $t(s)$ & $\boldsymbol{E}(\boldsymbol{w} \boldsymbol{t} \%)$ \\
\hline 1157 & 29,724 & 57.2 & 0.839 & $2.22 \times 10^{16}$ & $1.41 \times 10^{17}$ & 900 & 0.056 \\
\hline
\end{tabular}

$$
\begin{gathered}
N_{285}=\frac{A_{186}}{Y_{186} \exp \left(\frac{-\ln (2) t}{T_{\frac{1}{2} 285}}\right)} \approx \frac{A_{186}}{Y_{186}} \\
N_{288}=\frac{A_{1001}}{Y_{1001} \exp \left(\frac{-\ln (2) t}{T_{\frac{1}{2} 288}}\right)} \approx \frac{A_{1001}}{Y_{1001}} \\
E(w t \%)=\frac{235\left(N_{285}\right)}{235\left(N_{285}\right)+238\left(N_{288}\right)}
\end{gathered}
$$





\section{APPENDIX C. ESTIMATION OF URANIUM ENRICHMENT FROM 186-258 KEV GAMMA LINES}





\section{APPENDIX C. ESTIMATION OF URANIUM ENRICHMENT FROM 186-258 KEV GAMMA LINES}

The equations below use the following variables, with their values listed in Table C.1:

- $t$ (s) is the live time of the passive gamma spectroscopy measurement

- $A_{186}, A_{1001}$ are the net counts under the $186 \mathrm{keV}$ and $1001 \mathrm{keV}$ peaks, respectively

- $\quad T_{\frac{1}{2}, 235}, T_{\frac{1}{2}, 238}(\mathrm{~s})$ are the half-lives of ${ }^{235} \mathrm{U}$ and ${ }^{238} \mathrm{U}$, respectively

- $\quad Y_{186}, Y_{1001}$ are the fraction of decays that produce the $186 \mathrm{keV}$ and $1001 \mathrm{keV}$ peaks, respectively

- $\quad N_{235}, N_{238}$ are the number of ${ }^{235} \mathrm{U}$ and ${ }^{238} \mathrm{U}$ atoms

- $\quad E$ (wt \%) is the estimate of the ${ }^{235} \mathrm{U}$ enrichment

Table C.1. 186 and $258 \mathrm{keV}$ net peak areas, fraction of decays, and half-lives for ${ }^{235} U$ and ${ }^{238} U$ used to estimate enrichment of Object 1

\begin{tabular}{|c|c|c|c|c|c|c|c|}
\hline$A_{186}$ & $A_{258}$ & $\boldsymbol{Y}_{186}(\%)$ & $\boldsymbol{Y}_{258}(\%)$ & $\boldsymbol{T}_{\frac{1}{z} 235}(\mathrm{~s})$ & $\boldsymbol{T}_{\frac{1}{z} 238}(\mathrm{~s})$ & $\boldsymbol{t}(\boldsymbol{s})$ & $\boldsymbol{E}(\boldsymbol{w} t \%)$ \\
\hline 1157 & 2110 & 57.2 & $7.3 \times 10^{-2}$ & $2.22 \times 10^{16}$ & $1.41 \times 10^{17}$ & 900 & 0.066 \\
\hline
\end{tabular}

$$
\begin{aligned}
& N_{285}=\frac{A_{186}}{Y_{186} \exp \left(\frac{-\ln (2) t}{T_{\frac{1}{2} 285}}\right)} \approx \frac{A_{186}}{Y_{186}} \\
& N_{288}=\frac{A_{258}}{Y_{258} \exp \left(\frac{-\ln (2) t}{T_{\frac{1}{2} 238}}\right)} \approx \frac{A_{258}}{Y_{258}} \\
& E(w t \%)=\frac{235\left(N_{285}\right)}{235\left(N_{285}\right)+238\left(N_{288}\right)}
\end{aligned}
$$



APPENDIX D. DERIVATION OF FEYNMAN VARIANCE 



\section{APPENDIX D. DERIVATION OF FEYNMAN VARIANCE}

The equations below use the following variables:

- $\quad m_{1}, m_{2}$ are the measured singles and doubles counts, respectively

- $\quad y_{1}, y_{2}$ are the singles and doubles with random counts removed, respectively

- $\quad Y(T)$ is the Feynman variance

Please note the following definitions that are used in the derivation:

- $\left(\begin{array}{l}n \\ k\end{array}\right)=\frac{n !}{k !(n-k) !}$

- $\quad Y(T)=\left(\frac{\overline{c^{2}}-\bar{c}^{2}}{\bar{c}}-1\right)$

$$
\begin{gathered}
y_{1}=m_{1}=\sum_{j=1}^{3}\left(\begin{array}{l}
j \\
1
\end{array}\right) b_{j}=\sum_{j=1}^{3} j b_{j}=\bar{c} \\
y_{2}=m_{2}-\frac{y_{1}^{2}}{2 !}=\sum_{j=2}^{3}\left(\begin{array}{l}
j \\
2
\end{array}\right) b_{j}-\frac{y_{1}^{2}}{2}=\sum_{j=2}^{3}\left(\begin{array}{l}
j \\
2
\end{array}\right) b_{j}-\frac{\left(\sum_{j=1}^{\mathrm{g}} j b_{j}\right)^{2}}{2} \\
y_{2}=\sum_{j=2}^{3} \frac{j(j-1) b_{j}}{2}-\frac{\left(\sum_{j=1}^{\mathrm{g}} j b_{j}\right)^{2}}{2}=\frac{1}{2}\left(\sum_{j=2}^{3} j^{2} b_{j}-\sum_{j=1}^{3} j b_{j}-\left(\sum_{j=1}^{3} j b_{j}\right)^{2}\right) \\
y_{2}=\frac{1}{2}\left(c^{2}-\bar{c}^{2}-\bar{c}\right) \\
\frac{y_{2}}{y_{1}}=\frac{1}{2}\left(\frac{c^{2}-c^{2}}{\bar{c}}-1\right)=\frac{1}{2} Y(T) \\
\therefore Y(T)=2\left(\frac{y_{2}}{y_{1}}\right)
\end{gathered}
$$



APPENDIX E. ENRICHMENT CURVE FIT VALUES 



\section{APPENDIX E. ENRICHMENT CURVE FIT VALUES}

The line of best fit equation used to form the enrichment curves to determine the enrichment of the HEU present in Object 1 employs the following variables, with their values listed in Table E.1:

- $\quad N_{\text {integrated }}$ is the number of integrated reference counts on the TOF plots of pixels 5 through 12 over the window of 35 to $80 \mathrm{~ns}$

- $\quad E\left(\right.$ wt \%) is the estimate of the ${ }^{235} \mathrm{U}$ enrichment

- $\quad a, b$, and $c$ are constants determined by Matlab to best fit the plotted data

Table E.1. Integrated reference counts, $N_{\text {integrated }}$, and line of best fit constants, $a, b$, and $c$, used to interpolate to determine the enrichment, $E$, of the HEU in Object 1

\begin{tabular}{ccccc}
\hline Pixel number & $\boldsymbol{N}_{\text {integrated }}$ & $\boldsymbol{a}$ & $\boldsymbol{b}$ & $\boldsymbol{c}$ \\
\hline 5 & 7463 & 98.81 & -77.09 & 1220.84 \\
6 & 8948 & -136.90 & 491.89 & 2448.59 \\
7 & 8957 & -21.27 & 807.05 & 3644.96 \\
8 & 8861 & 1480.86 & -504.12 & 4926.66 \\
9 & 8783 & 1350.36 & 1644.52 & 5248.53 \\
10 & 8944 & 1366.58 & 2523.02 & 5750.99 \\
11 & 8819 & 2912.10 & 898.96 & 6043.78 \\
12 & 7316 & 3010.97 & 771.00 & 5928.31 \\
\hline
\end{tabular}

$N_{\text {integrated }}=a E^{2}+b E+c$ 
